\title{
A Survey of Floor Vibration Noise at all Sectors in the APS Experiment Hall
}

\author{
Steven Kearney ${ }^{1}$ and Deming Shu ${ }^{1}$ \\ ${ }^{1}$ X-ray Science Division, APS, Argonne National Laboratory, Argonne, IL. 60439
}

\section{ANL/APS/LS-344}

\begin{abstract}
A vibration survey of the APS experiment hall floor was conducted. It was found that beamlines $\mathbf{1 0 - 2 0}$ have particularly low levels of vibration when compared to the rest of the facility. The vibration spectrum for each beamline floor can be found in the appendix. Throughout the majority of the $5-100 \mathrm{~Hz}$ vibration spectrum beamlines at the APS fall below the most stringent NEST vibration criteria. Lastly, it was concluded that the magnitude of vibrations at a particular beamline is largely dependent upon the magnitude of vibrations present at the nearby mezzanine support column.
\end{abstract}

\section{Introduction}

Vibration noise in the experiment hall of the Advanced Photon Source (APS) is a potential primary source of mechanical noise induced into experiments being conducted at the APS. Therefore, understanding the current levels of vibration and locations of particularly low and high vibrations is of upmost importance. In addition, with the planned APS upgrade new beamline construction and/or redesigns of existing beamlines is expected, so it is beneficial to know the current levels of vibration at these locations for planning purposes. This survey was conducted at each beamline of the APS and presents the findings with a brief analysis of potential vibration sources as well as identifying locations of low vibration noise.

\section{Procedure}

A vibration noise survey was conducted at the Argonne National Laboratory Advanced Photon Source experiment floor. Data was recorded at all beamlines over multiple sessions. For consistency, measurements were only taken the day before the scheduled weekly machine intervention when most users were not running experiments, but with the beam still on. Also, to limit the influence of transient vibrations, data was recorded only after $5 \mathrm{pm}$ or on the weekends. The specific dates of measurement sessions were: 3/28/2016, 4/4/2016, 4/11/2016, 4/18/2016, 4/25/2016, and 6/12/2016. In addition, a separate special case data set was recorded in sector 21 during electrical maintenance (4/30/2016) in which the mechanical air handling unit, DI pumps, 
and vacuum pumps, were shut down in sectors 20-23. This data was then used to assess the contribution of these systems to the vibration noise in sector 21 . This is just a single sector comparison, but due to the modular similarity of mechanical equipment around the storage ring this can be assumed to represent typical mechanical noise contributions for all beamlines.

To measure the vibration noise, 3 high sensitivity accelerometers were used (VibraMetrics 1030, Mistras Group Inc., Princeton Junction, NJ) for each Cartesian direction, see Table 1. In line with the accelerometers were 3 power supplies for each channel (VibraMetrics P5000, Mistras Group Inc., Princeton Junction, NJ). For data acquisition a Photon+ 4 channel (Brüel \& Kjær) signal analyzer was used with RT Pro Version 7.20 dynamic signal analysis software (Brüel \& Kjær). The data acquisition settings can be seen in Table 2.

Table 1. Specifications and settings of accelerometers used for each channel recorded. See Figure 1 for specific channel location and coordinate frame.

\begin{tabular}{lccccc}
\hline Channel & Direction & Model No. & Serial No. & Gain & Sensitivity [V/g] \\
\hline 1 & $\mathrm{X}$ & VibraMetrics 1030 & 1349 & $\mathrm{x} 1$ & 7.088 \\
2 & $\mathrm{Y}$ & VibraMetrics 1030 & 1625 & $\mathrm{x} 1$ & 7.000 \\
3 & $\mathrm{Z}$ & VibraMetrics 1030 & 1493 & $\mathrm{x} 1$ & 7.010 \\
\hline
\end{tabular}

Data was acquired separately at all 35 beamlines of the APS. Two measurement points for each beamline were chosen based on similar construction geometry and for the best representation of vibrations for the entire beamline. These two points can be seen in Figure 1. The column floor point is assumed to represent the majority of the noise source to the floor for the nearest beamline. This assumption comes from the fact that the column is a support structure for the mechanical equipment mezzanine floor. There are many more columns than there are beamlines so the column closet to the floor measurement point was chosen, see Table 3 for the specific column measured and which beamline it represents. Similarly, the beamline floor point is assumed to represent the noise present for that entire beamline. Notice that the red dashed line shows many potential measurement points. This is required due to the varying construction designs and surrounding equipment layout of each column and end station.

Table 2. Parameter settings used in the RT Pro 7.2 dynamic signal analysis software for data acquisition.

\begin{tabular}{lcc}
\hline Parameter & Value & Unit \\
\hline Sampling Frequency & 375 & $\mathrm{~Hz}$ \\
Number of Samples & 4096 & \\
Bandwidth & 150 & $\mathrm{~Hz}$ \\
Frequency Resolution & 0.091 & $\mathrm{~Hz}$ \\
Window & Hanning & \\
Averages & 20 & \\
\hline
\end{tabular}


For analysis, the data is presented in spectral RMS displacement and in mean RMS displacement for individual octave bands. RMS displacement is derived from the raw accelerometer voltage data using

$$
U_{r m s}=\frac{g V_{r m s}}{\text { (gain)(sensitivity) }}, \text { with } g=9.81 \mathrm{~m} / \mathrm{s}
$$

The mean RMS displacement from defined frequency bands, adapted from (Rogers et al. 1997), is defined as

$$
\overline{U_{k}}=\frac{1}{N_{k}} \sum_{i=f_{\text {low }}(k)}^{f_{\text {high }}(k)} U_{r m s}(i),
$$

with $k$ defined as the band number, $i$ representing each frequency bin, and $N_{k}$ the number of bins in band $k$. In Equation (2) the units are still in RMS displacement with the RMS subscript removed for clarity. Presenting the data in bands rather than full spectral plots allows for a quick and concise comparison of a single value for each beamline. For this study standard octave bands were used and are listed in Table 4 as well as the number of frequency bins used in each band to calculate the mean.
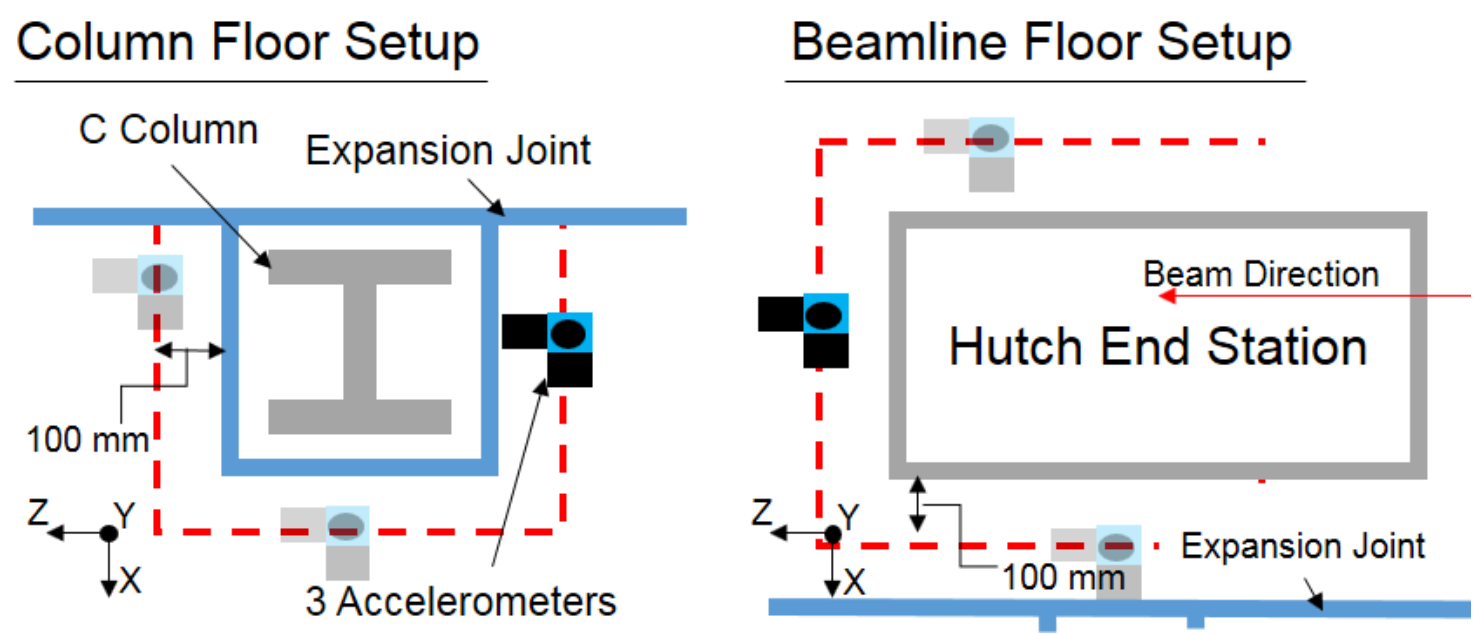

Figure 1. A bird's eye view of the two measurement points used for each beamline (not to scale). On the left is the setup for the column floor measurement point and on the right the beamline floor measurement point. For both, the coordinate frame is shown in the lower left with $X$ away from the storage ring center, $Y$ in the vertical direction, and $Z$ in the beam direction. The hutch end station is located in the $-X$ direction on the opposite side of the expansion joint of the column. The red dashed line represents potential placement locations of the sensor block, with the transparent blocks showing other potential locations, based on accessibility to the floor. 
Table 3. Beamlines and the associated nearby column that was measured.

\begin{tabular}{cccccc}
\hline Beamline & Column & Beamline & Column & Beamline & Column \\
\hline 1 & C069C & 13 & C104C & 25 & C140C \\
2 & C072C & 14 & C106C & 26 & C143C \\
3 & C074C & 15 & C110C & 27 & C145C \\
4 & C078C & 16 & C113C & 28 & C149C \\
5 & C081C & 17 & C116C & 29 & C152C \\
6 & C083C & 18 & C119C & 30 & C154C \\
7 & C086C & 19 & C121C & 31 & C159C \\
8 & C090C & 20 & C125C & 32 & C162C \\
9 & C093C & 21 & C129C & 33 & C165C \\
10 & C095C & 22 & C131C & 34 & C167C \\
11 & C098C & 23 & C135C & 35 & C169C \\
12 & C101C & 24 & C137C & & \\
\hline
\end{tabular}

Table 4. Octave bands used in the comparison of beamlines with upper and lower frequency bounds listed and the number of frequency bins averaged, $N_{k}$.

\begin{tabular}{lccc}
\hline Octave Band $k$ & $f_{\text {low }}[\mathrm{Hz}]$ & $f_{\text {high }}[\mathrm{Hz}]$ & $N_{k}$ \\
\hline 1 & 2.8 & 5.5 & 30 \\
2 & 5.5 & 11 & 62 \\
3 & 11 & 22 & 121 \\
4 & 22 & 44 & 241 \\
5 & 44 & 88 & 482 \\
\hline
\end{tabular}

\section{Results and Discussion}

\subsection{Experiment Hall Floor Vibration Levels}

Each beamline was individually measured at two points, the floor next to the nearest column and the beamline floor just outside the end station hutch. Full $X, Y, Z$ spectrums of vibration displacement for each beamline floor can be seen in the Appendix. Every individual beamline floor vibration spectrum (designated by the ID \# in the title over each plot) displays the beamline in a bold black line, and, for comparison, the maximum/minimum, and mean of all APS beamline floor vibrations are plotted in solid gray and red dash dot lines, respectively. From these spectrums a trend starts to appear; a trend that beamlines close to each other tend to have similar levels of noise. This trend will be much more clear when we look at band data in the next section. 
We can also use the entire set of beamline floor data to see the magnitude of vibrations in the APS experiment hall as a whole. Figure 2, displays the $X, Y, Z$ spectral vibration magnitude range of all the beamlines. Included in this plot are a few of the most stringent standard vibration criteria (VC) created by the Institute of Environmental Sciences and Technology (IEST) (Amick et al. 2005). Each curve is subsequently more sensitive and thus harder to achieve, with VC-E at $3.2 \mu \mathrm{m} / \mathrm{s}, V C-F$ at $1.56 \mu \mathrm{m} / \mathrm{s}$, and VC-G at $0.78 \mu \mathrm{m} / \mathrm{s}$. VC-E, is described as "Challenging to achieve ... Assumed to be adequate for the most demanding of sensitive systems.", with an achievable detail size in microelectronics fabrication of less than $100 \mathrm{~nm}$. As can be seen in Figure 2 almost all of the floor vibrations are below the VC-E curve with the exception being the maximum line at $30 \mathrm{~Hz}$. Even more encouraging is that the majority of vibration magnitudes are below the most stringent VC-G curve with only 5 peaks in the maximum line breaking the VC-G curve at $15 \mathrm{~Hz}, 18 \mathrm{~Hz}, 23 \mathrm{~Hz}, 30 \mathrm{~Hz}$, and $60 \mathrm{~Hz}$. Overall the entire APS experiment hall floor is an extremely quiet facility and a very good starting point to build the most sensitive equipment on. However, there is still room for improvement, particularly in the $10-60 \mathrm{~Hz}$ band, which will be made more clear in Section 3.3. 

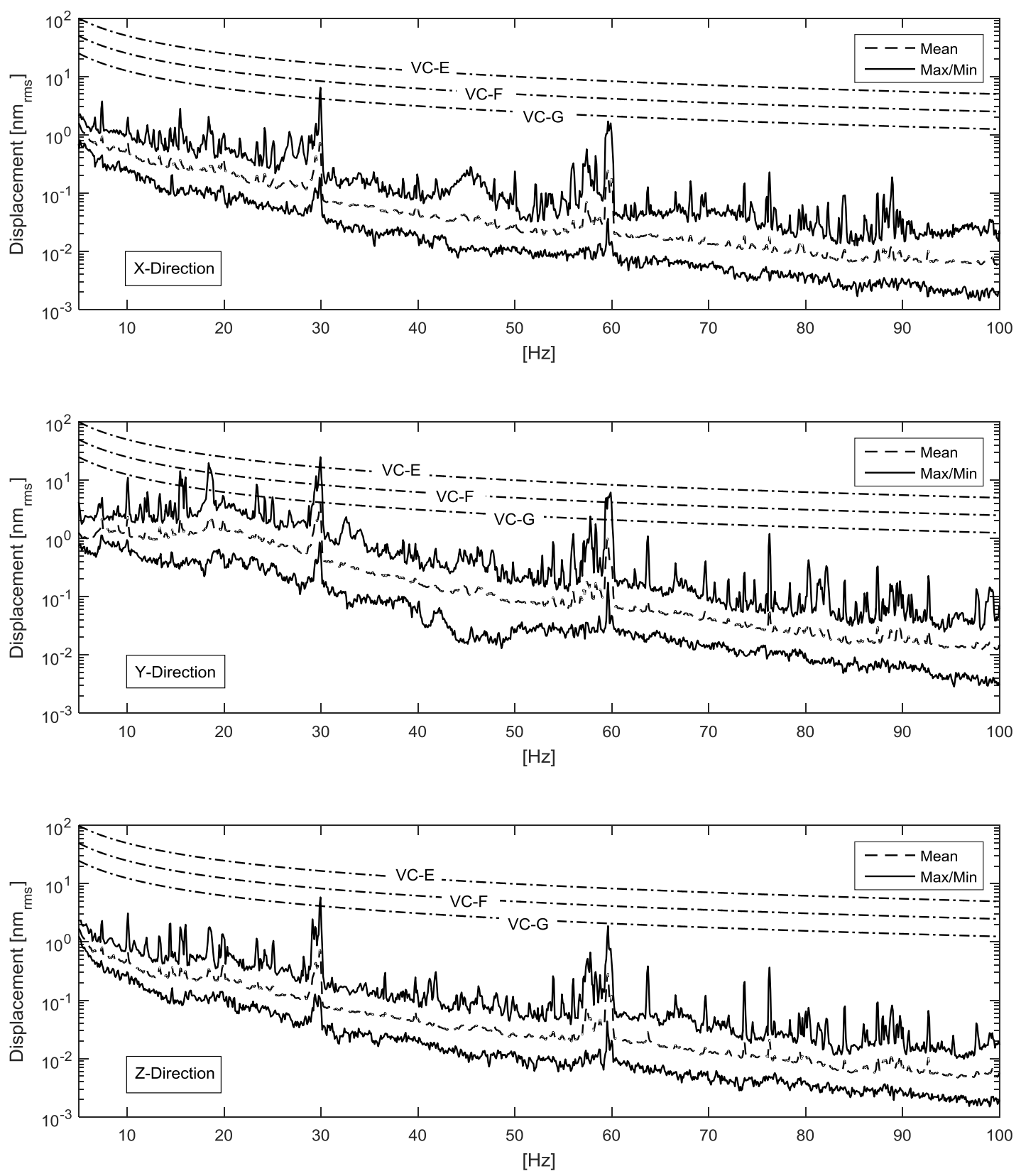

Figure 2. Vibration displacement spectrum of the entire experiment hall for all beamline floors in all 3 directions. The dashed line is the mean of all beamline floors, the solid black line represents the maximum and minimum vibrations of all beamlines, and VC curves are shown with dash dot lines. 


\subsection{Comparison of Beamline Vibration Bands}

One of the goals of this survey was to elucidate locations in the APS experiment hall that are particularly quiet. Using mean RMS displacement data for a particular octave band assigns a single scalar magnitude to a beamline for that band, which makes direct comparison of beamline to beamline much more straight forward. Figures 3-5, display the 5 octave bands for each beamline in the $\mathrm{X}, \mathrm{Y}$, and $\mathrm{Z}$ directions, respectively. Also included are the mean RMS displacement vibrations for the columns as well.

From the band plots two trends can be seen. The first trend is that the higher the vibration magnitude in the column floor the higher the vibration magnitude of the beamline floor. This can be seen by following the trend of the column curve which then mirrors the beamline curves, particularly in the octave bands 1-3. In octave bands 4 and 5 we start to see a separation in magnitude, which makes sense as higher frequency vibrations dampen at a much greater rate than low frequency vibrations. However, even with the separation in magnitude the trends of column and beamline seem to still have the same general profile.

The second trend is that there is a clear region of the experiment hall that has particularly low magnitude vibrations. This region is located approximately in beamlines 10-20. However, in the bands 4 and 5 there seems to be a greater variation from beamline to beamline. Even beamlines right next to each other can have much higher vibration magnitudes, which is also reflected in the columns. This might be in direct response to a particularly noisy localized area in the mechanical mezzanine floor. There are also two major outlies. In the maximum extreme is beamline 29, and it is clear that column 152 nearby 29-ID is also particularly noisy. So, it is likely that the high vibrations at 29 are from some noisy source on the mezzanine nearby. On the other hand, is the minimum extreme, and this is located at beamline 16, especially in the $Y$ direction. Beamline 16-ID is unique in that the floor is actually a bridge over a road access tunnel. It is possible that being constructed on a bridge structure the floor is more isolated from the lower frequency noise associated with ground motion and traffic, and that noise from the mechanical equipment on the mezzanine floor has a longer direct path to the beamline floor allowing for greater damping of vibrations by the time they reach the measurement point. 

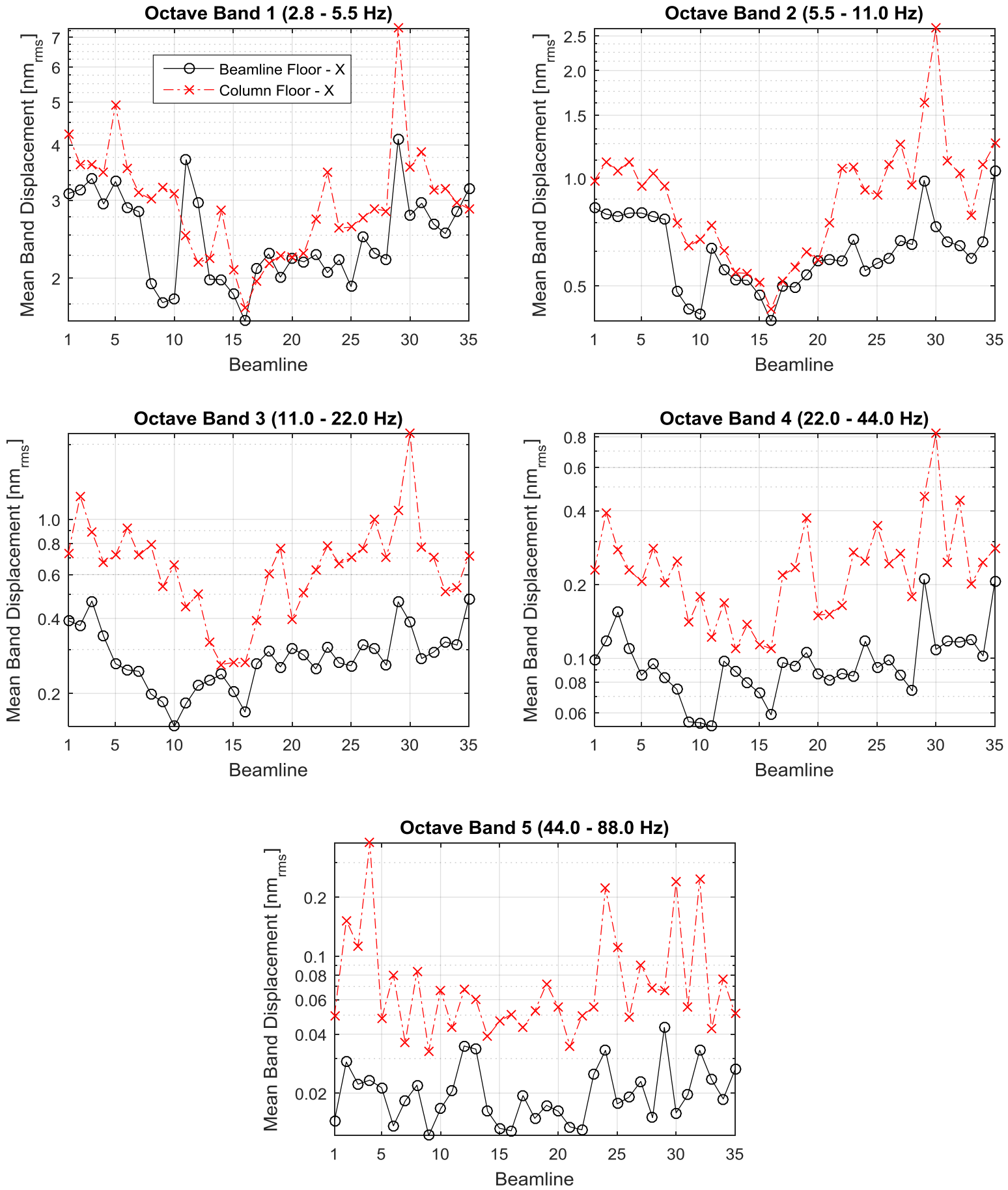

Figure 3. Mean displacement vibration for all beamlines in the $\mathrm{X}$ direction for each octave band. 

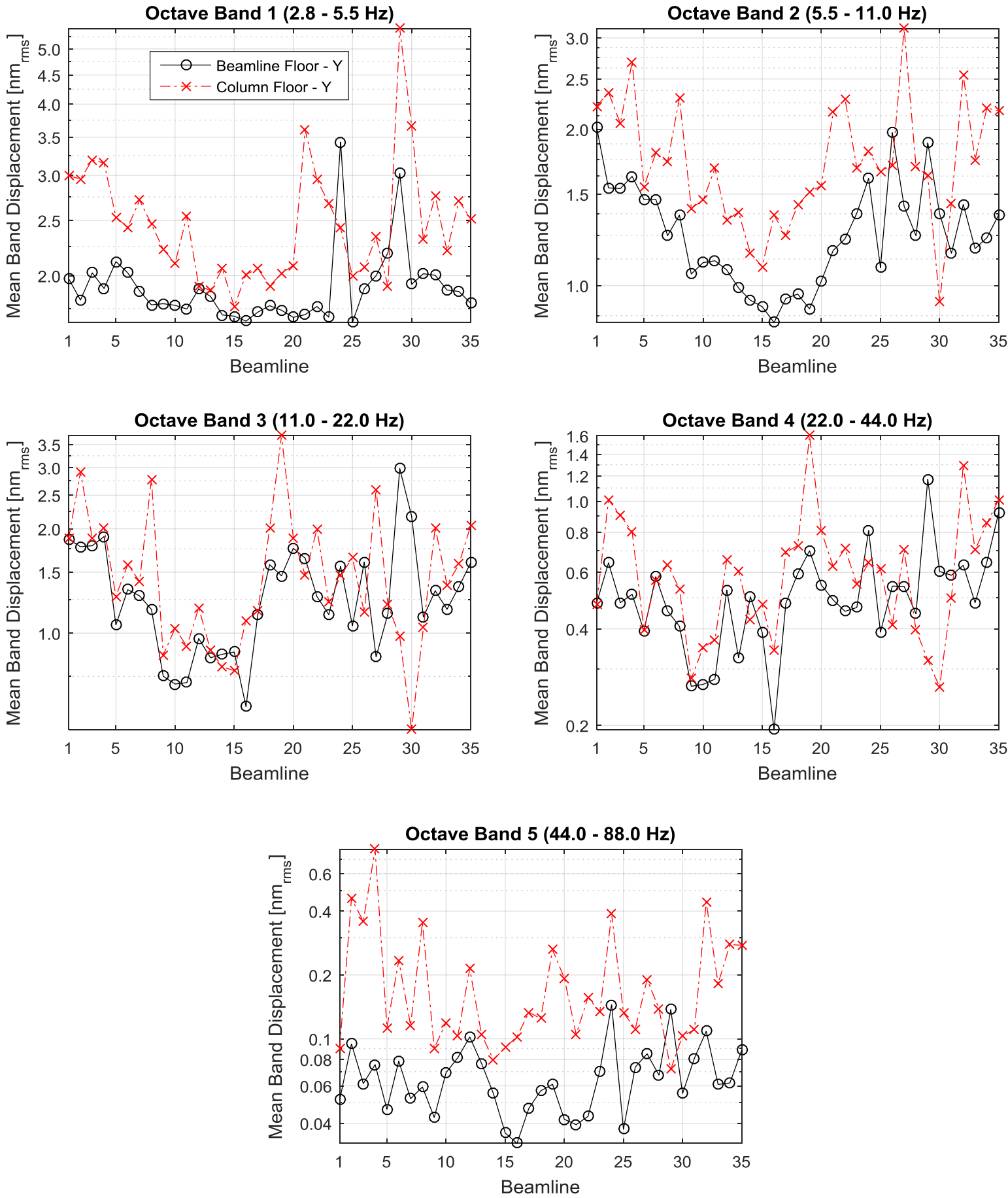

Figure 4. Mean displacement vibration for all beamlines in the $Y$ direction for each octave band. 

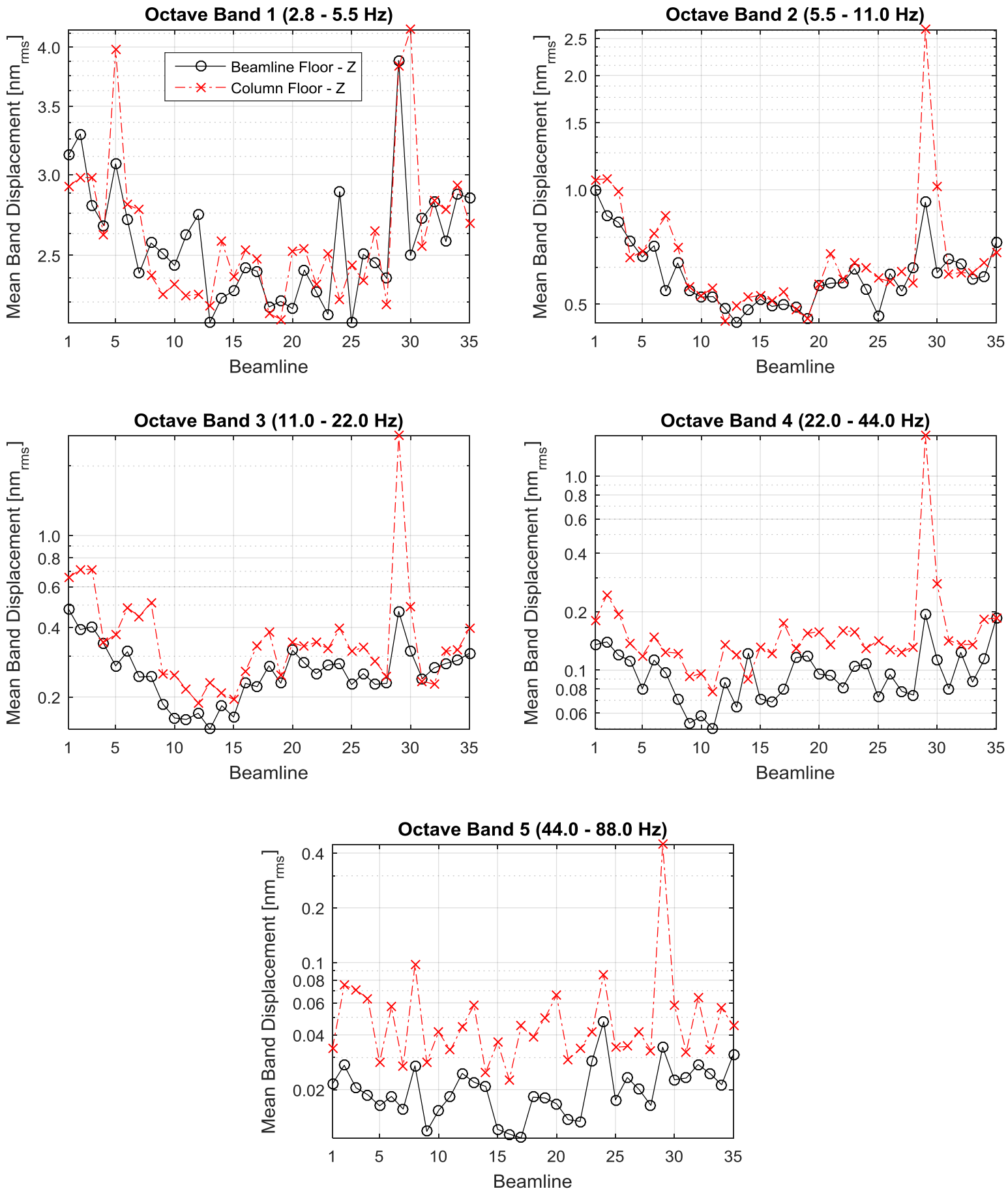

Figure 5. Mean displacement vibration for all beamlines in the $Z$ direction for each octave band. 


\subsection{Contribution of Mechanical Equipment to Noise}

In addition to the survey of floor vibrations a unique opportunity presented itself to measure the floor vibrations while the power to mechanical equipment was off. Vacuum pumps, DI pumps, and air handling equipment, all located on the mezzanine floor, were shut off for electrical maintenance in sectors 20-23. The same measurement points as when power was on during the initial survey was then measured again during the shutdown for beamline 21 .

First, looking at the difference in column floor vibrations with power on and off, see Figure 6 , we can see that there is a noticeable reduction in vibration magnitude throughout much of the spectrum. This is particularly evident in the $\mathrm{X}$ direction, and in the $\mathrm{Y}$ direction the reduction seems to be localized to the $10-60 \mathrm{~Hz}$ range. The $10-60 \mathrm{~Hz}$ range makes sense as this is the range in which the equipment normally produces vibration noise. There is almost no visible reduction in the $Z$ direction, which suggests that the column is stiffer in that direction.

Now looking at 21-ID beamline floor vibrations, Figure 7, we can similar reductions across the spectrum as was seen in the column. The reduction in the $Y$ direction is, in this case throughout the entire spectrum, however the largest reduction is still in the $10-60 \mathrm{~Hz}$ range. In this case the $10-60 \mathrm{~Hz}$ reduction is seen in all three directions, which is likely from a combination of vibrations of multiple columns from varying angles to the measurement point contributing to the vibration noise. From this data it can be concluded that the columns represent a significant source, approximately a half order of magnitude increase of vibrations at various frequencies, and any reduction in vibration noise to mechanical equipment on the mezzanine floor should have a measured reduction in vibrations on the beamline floor. 

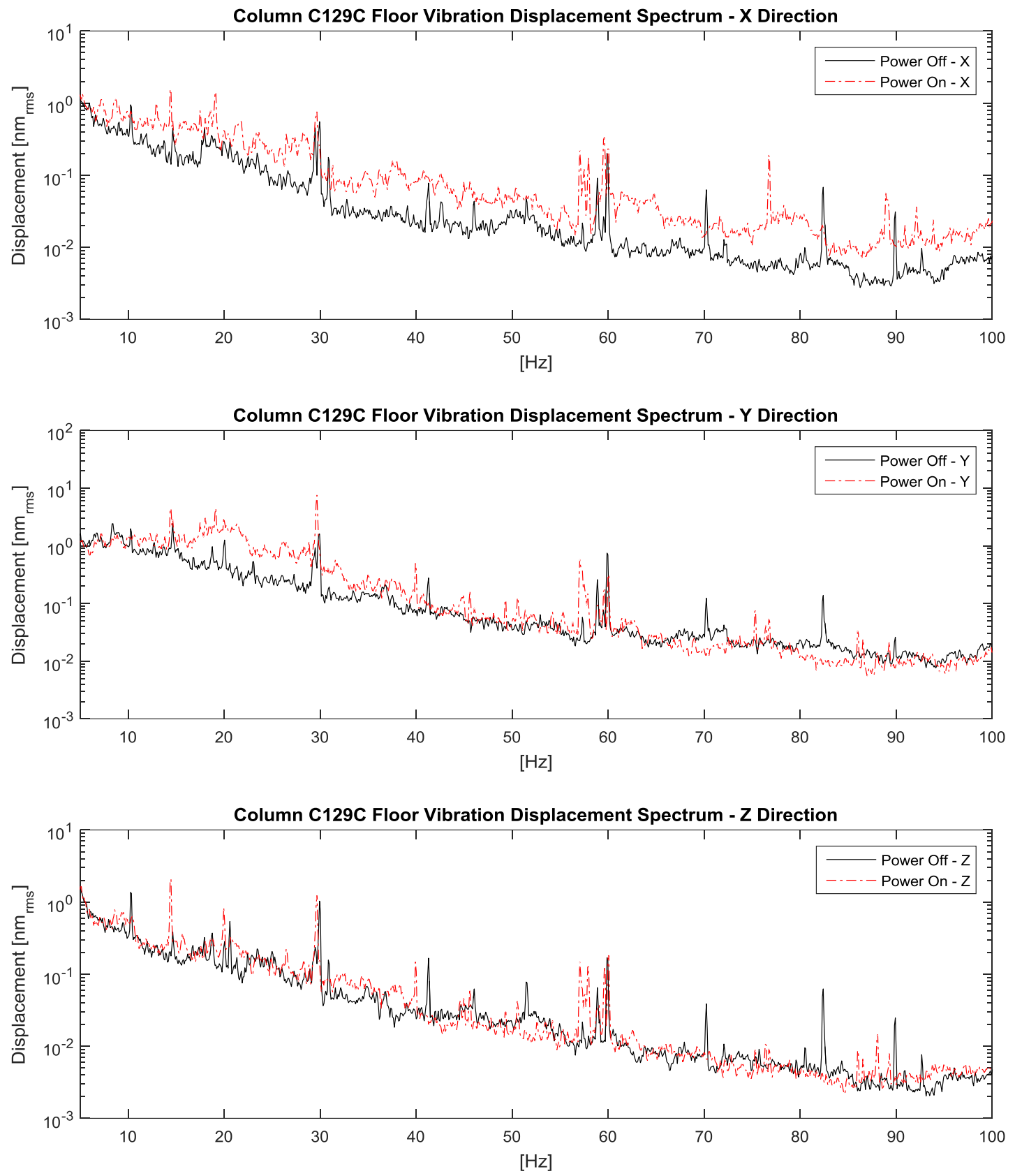

Figure 6. Comparison of column $\mathrm{C} 129 \mathrm{C}$ floor vibration displacement spectrum with the power on and power off to the mechanical equipment on the mezzanine. 

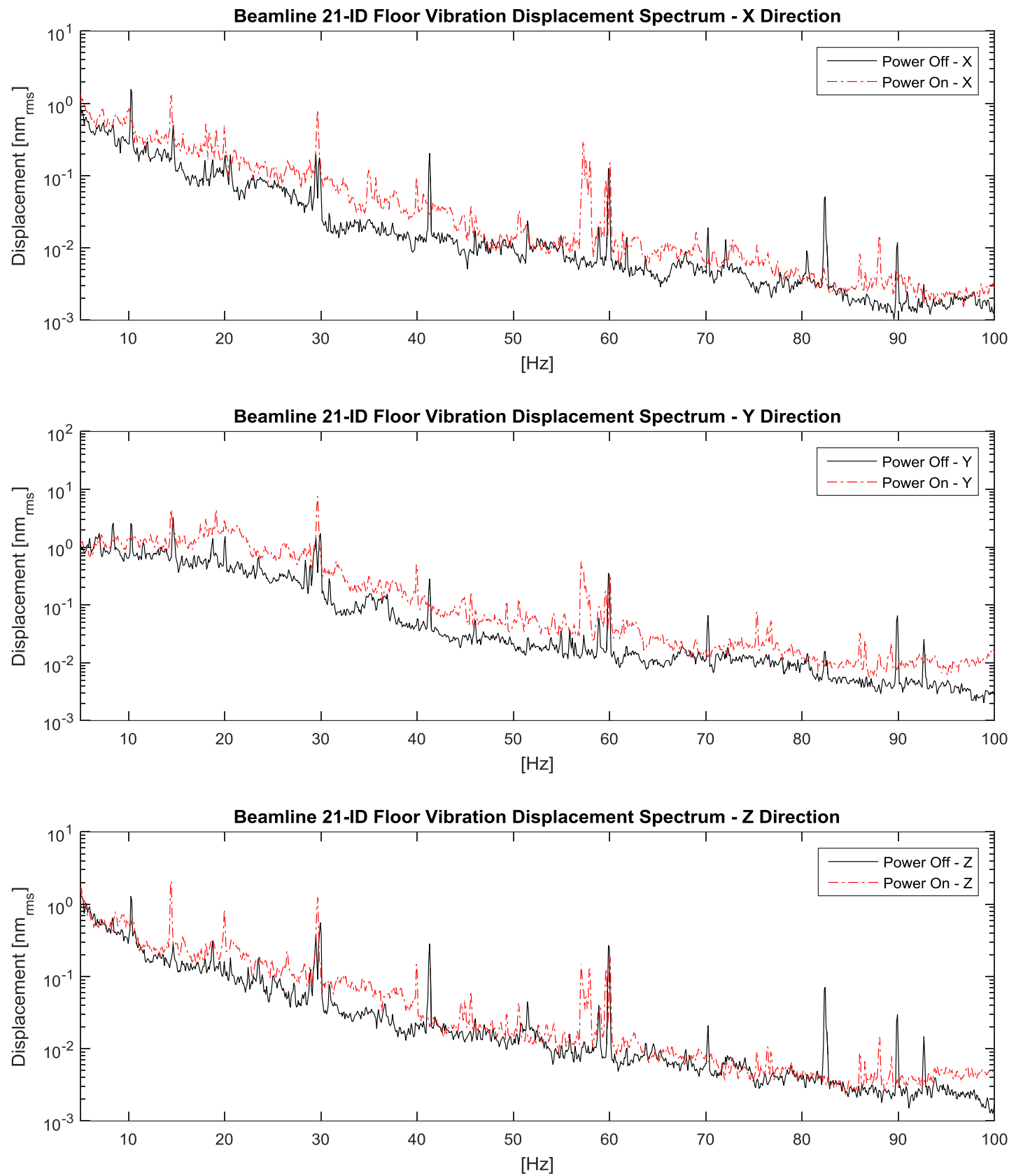

Figure 7. Comparison of the beamline floor vibration displacement spectrum with the power on and power off to the mechanical equipment on the mezzanine. 


\section{Conclusions}

A survey of the experiment hall at the APS was conducted and several conclusions can be gained from this survey. It was found that the entire APS experiment hall is an extremely vibration quiet research facility when compared to the VC curves from NEST. Even though, it was found that some regions of the floor have even lower magnitude vibrations than others, particularly beamlines 10-20. In the lower frequency bands, up to $22 \mathrm{~Hz}$, local similarity of vibration magnitudes could be seen, however above $22 \mathrm{~Hz}$ variation was seen from one beamline to the next. It was found that the column vibrations mirrored those of the nearby beamlines, and when the power was turned off to the mechanical equipment on the mezzanine the affected column and beamline floor had visible reductions in vibration magnitudes. This all points to the columns being a primary conveyor and source of vibration noise to the experiment hall floor. It is hoped that the results and conclusions of this survey will be used in support of the selection of future beamline construction areas, as a planning tool for future beamline designs, and to help identify sources of vibration noise.

\section{Acknowledgements}

The authors would like to acknowledge Dean Haeffner and Patricia Fernandez for their management and support of this project, and also Curt Preissner for his advice and professional insight into the project. Work supported by the U.S. Department of Energy, Office of Science, under Contract No. DE-AC02-06CH11357.

\section{References}

Amick, Hal, Michael Gendreau, Todd Busch, and Colin Gordon. 2005. "Evolving criteria for research facilities: vibration." Optics \& Photonics 2005.

Rogers, Melissa JB, Kenneth Hrovat, Kevin McPherson, Milton E Moskowitz, and Timothy Reckart. 1997. "Accelerometer data analysis and presentation techniques." 


\section{Appendix - Complete Beamline Floor Data Set}
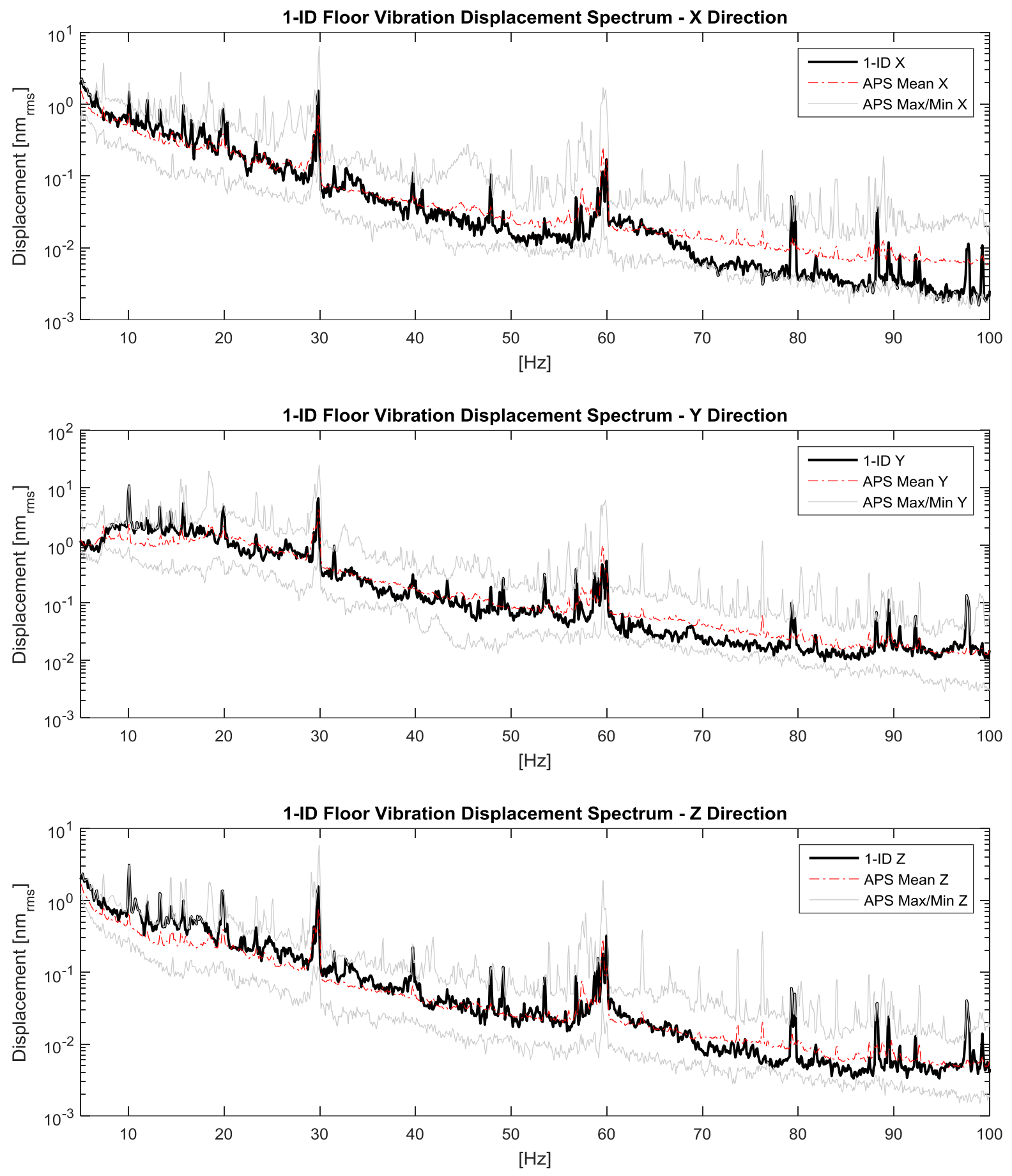

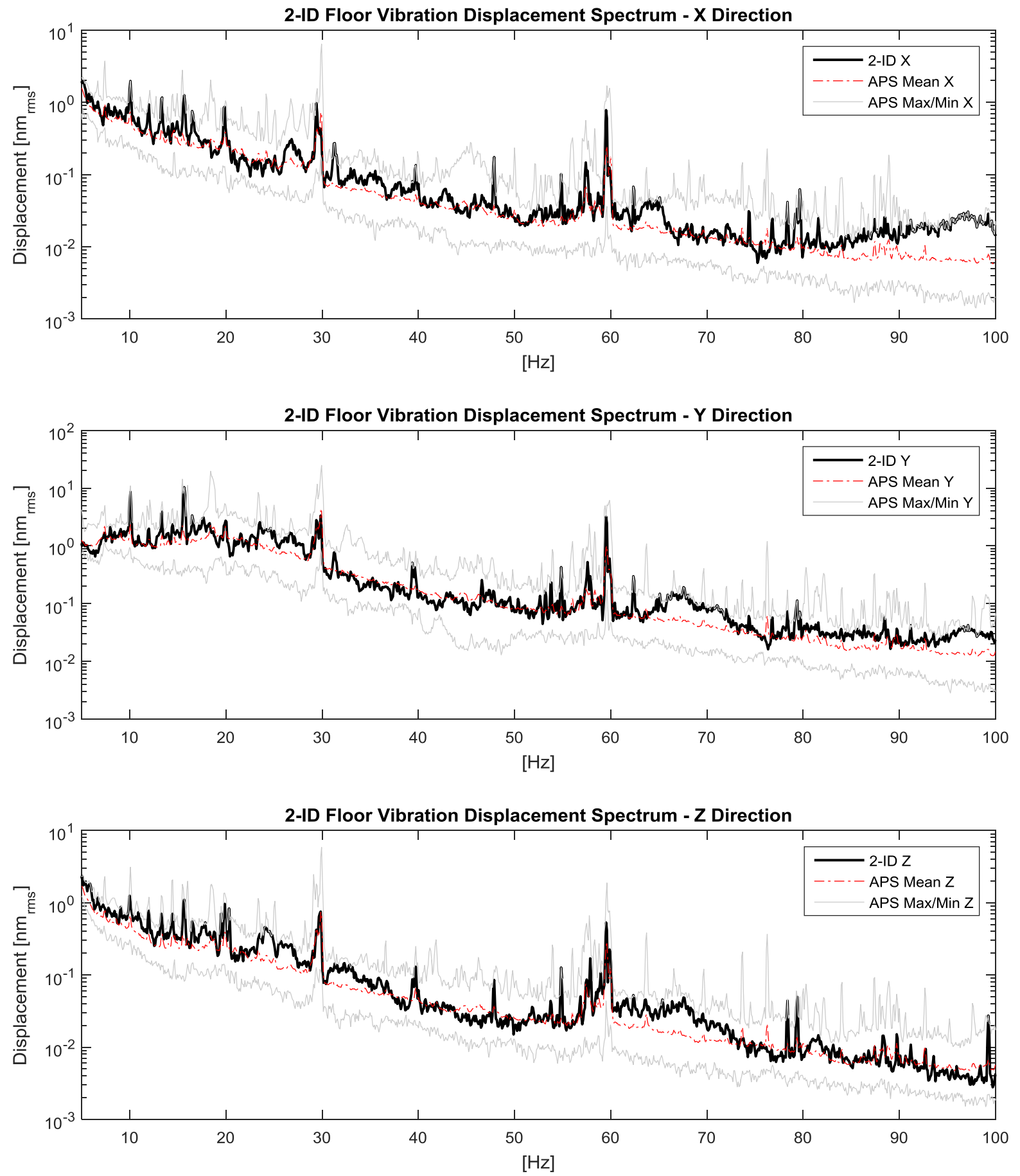

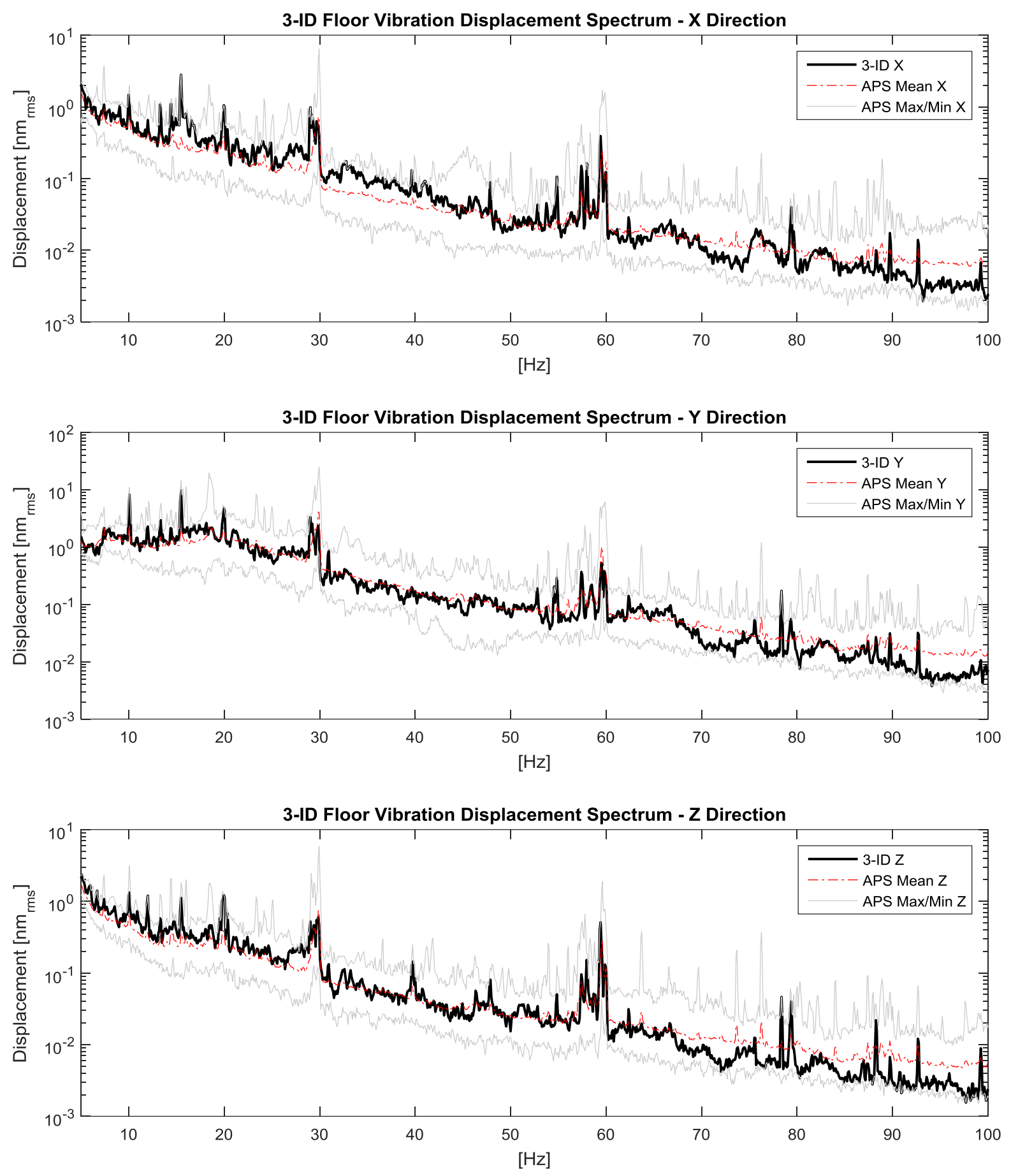

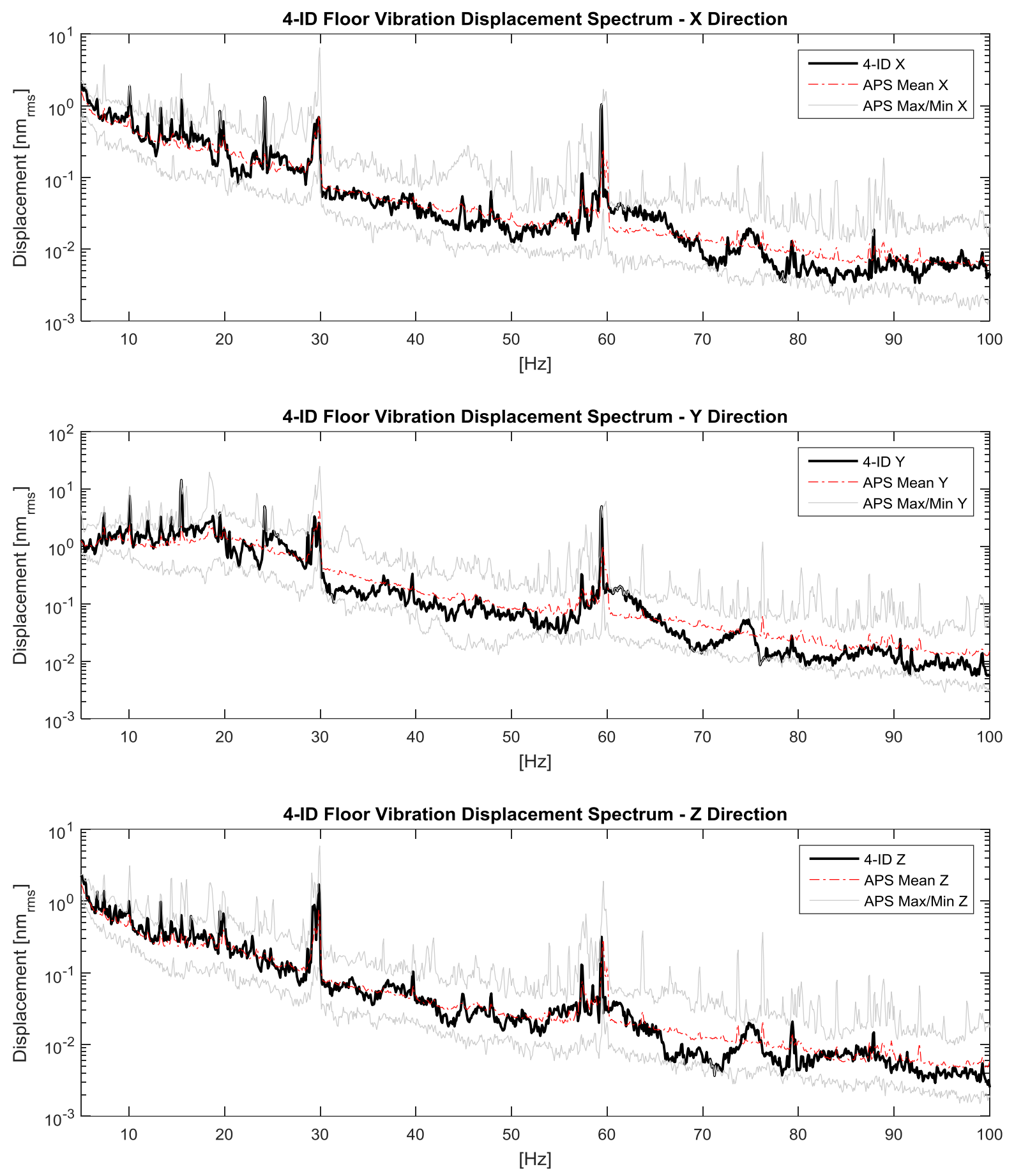

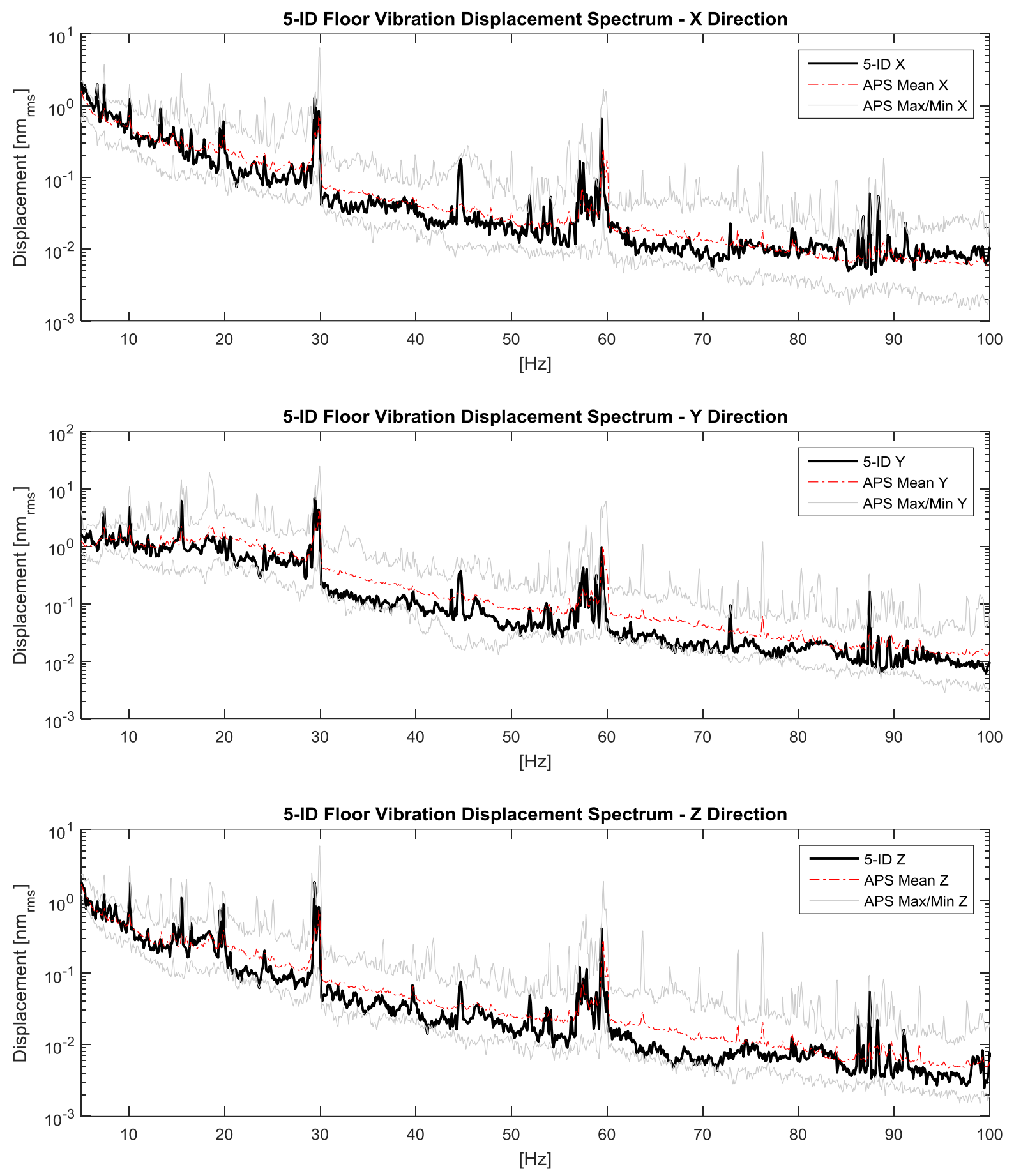

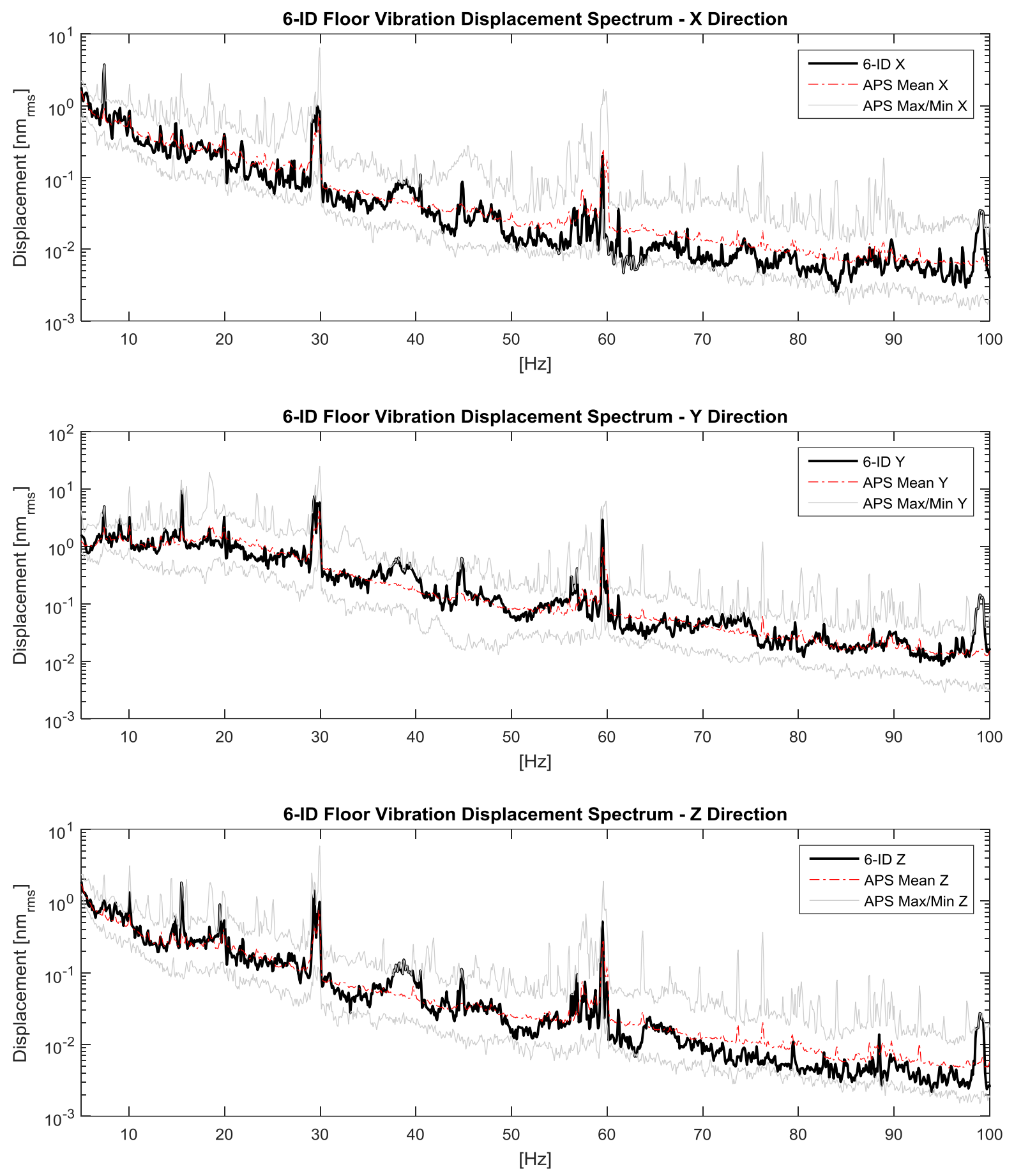

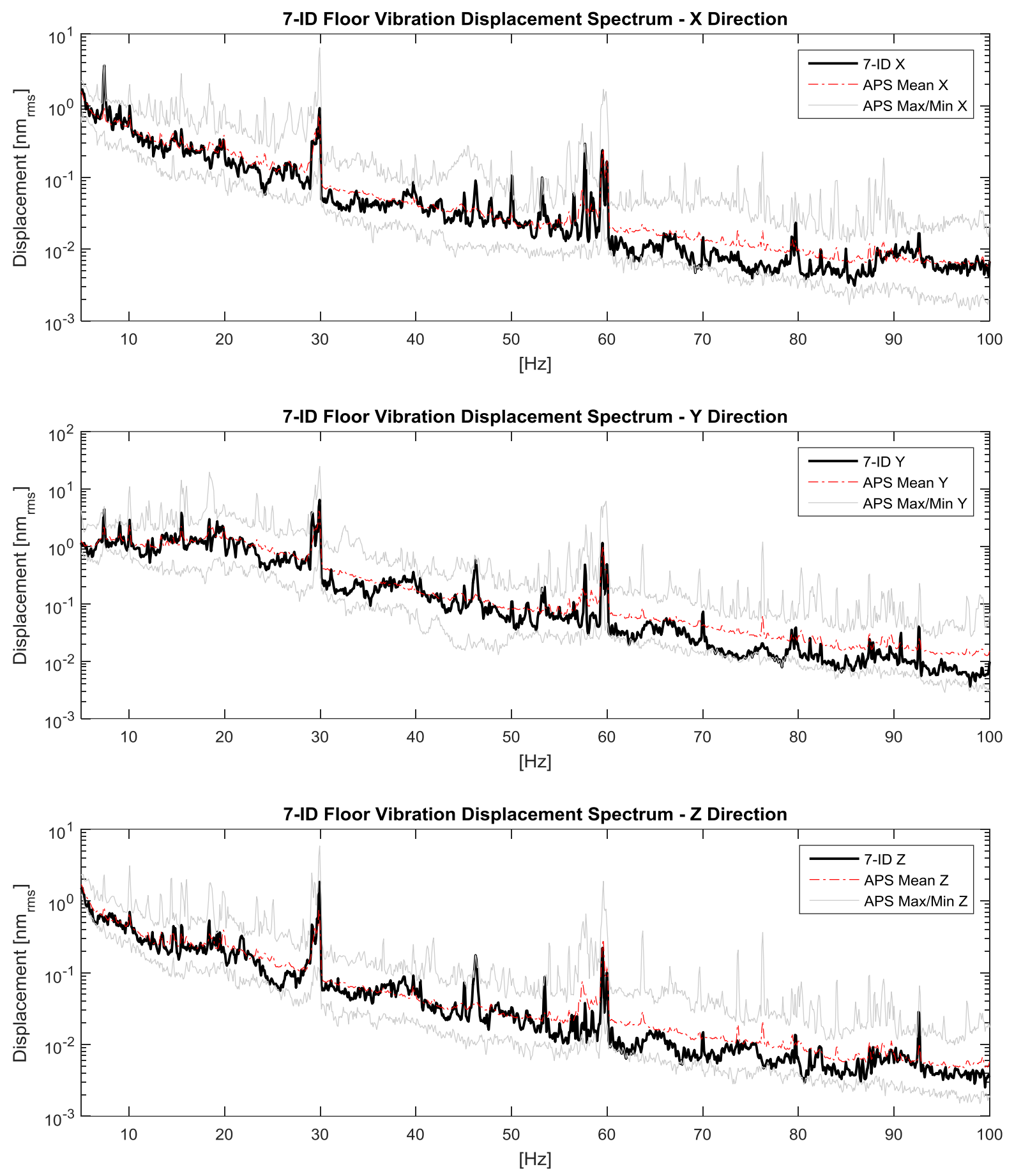

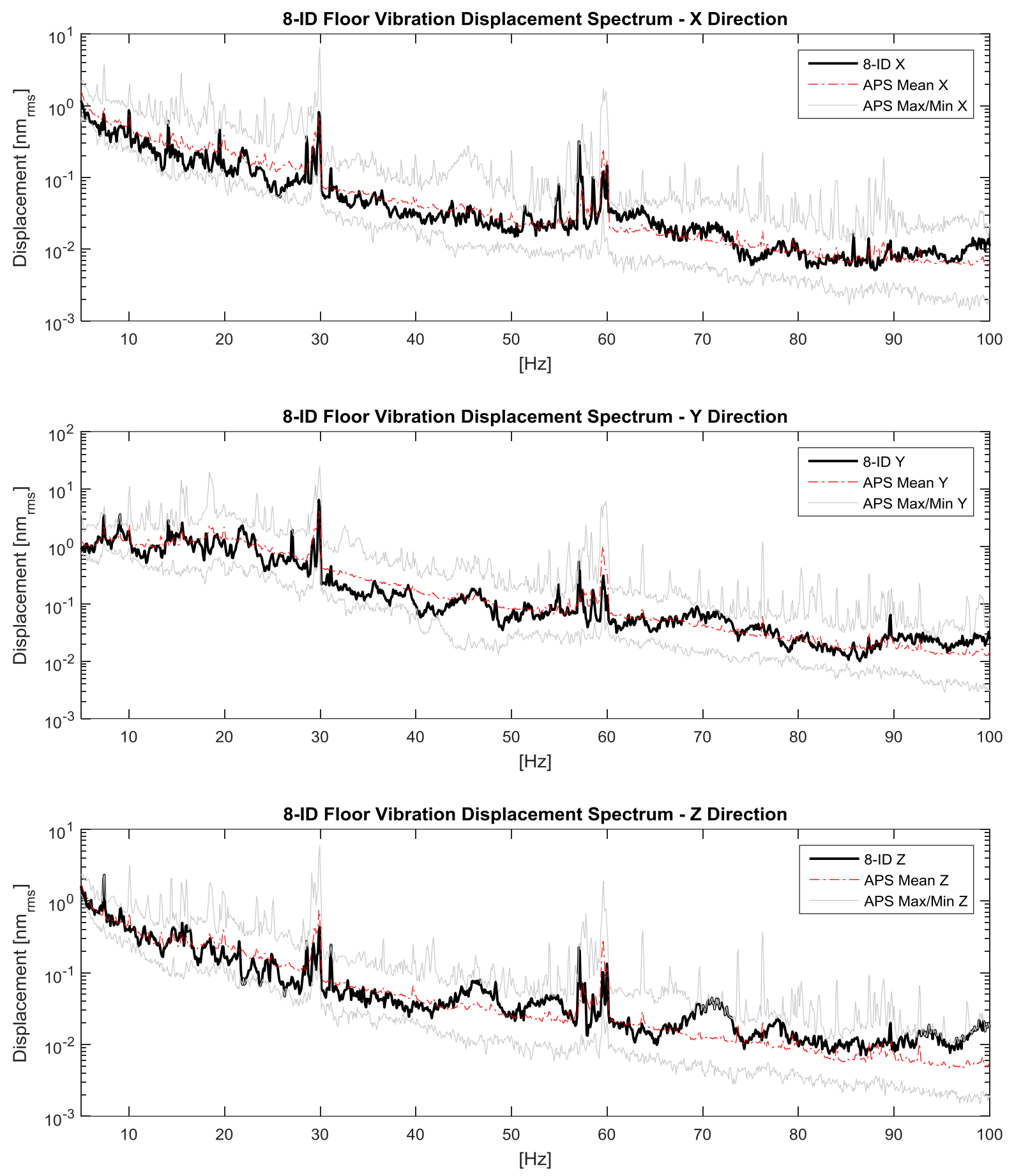

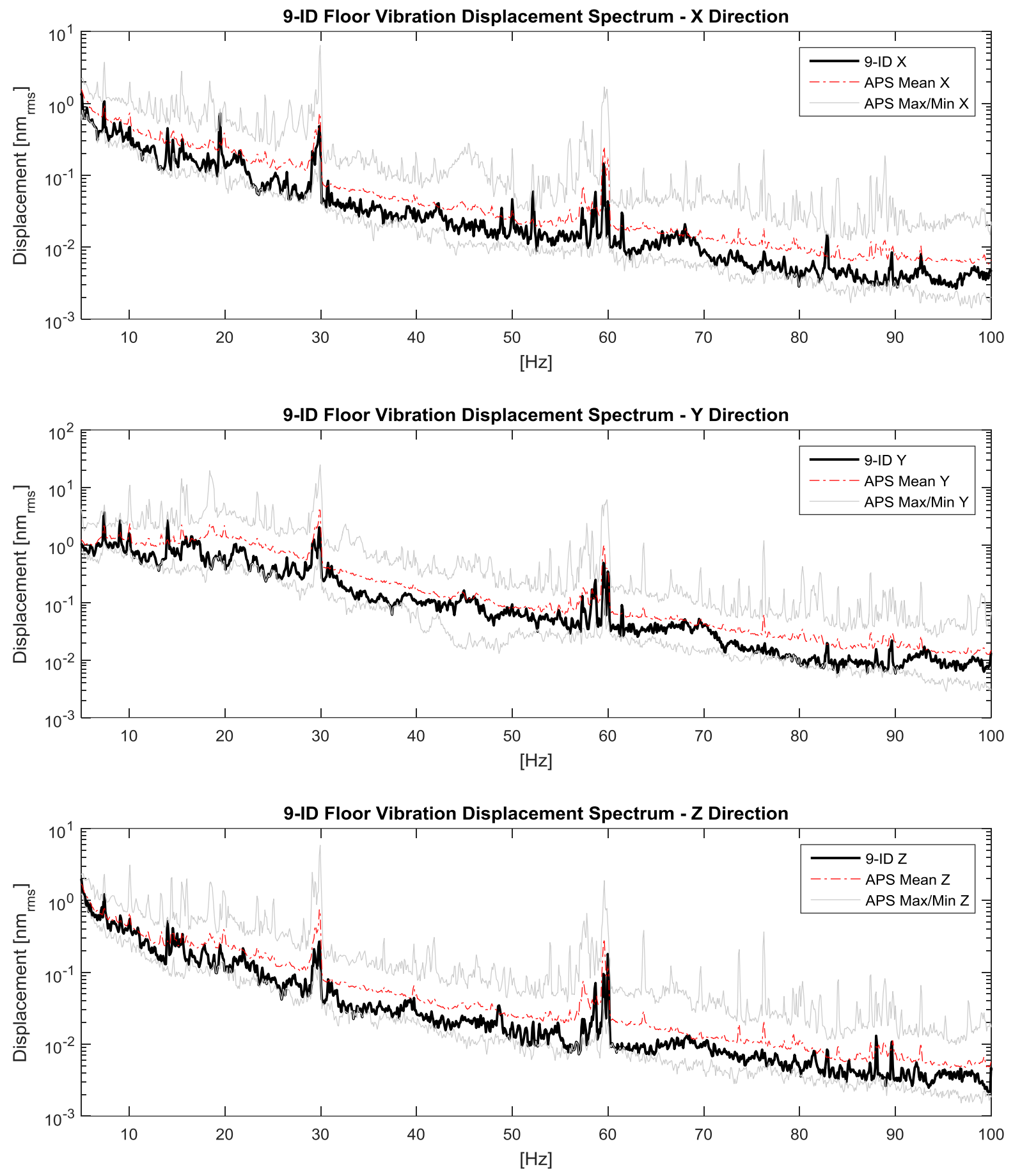

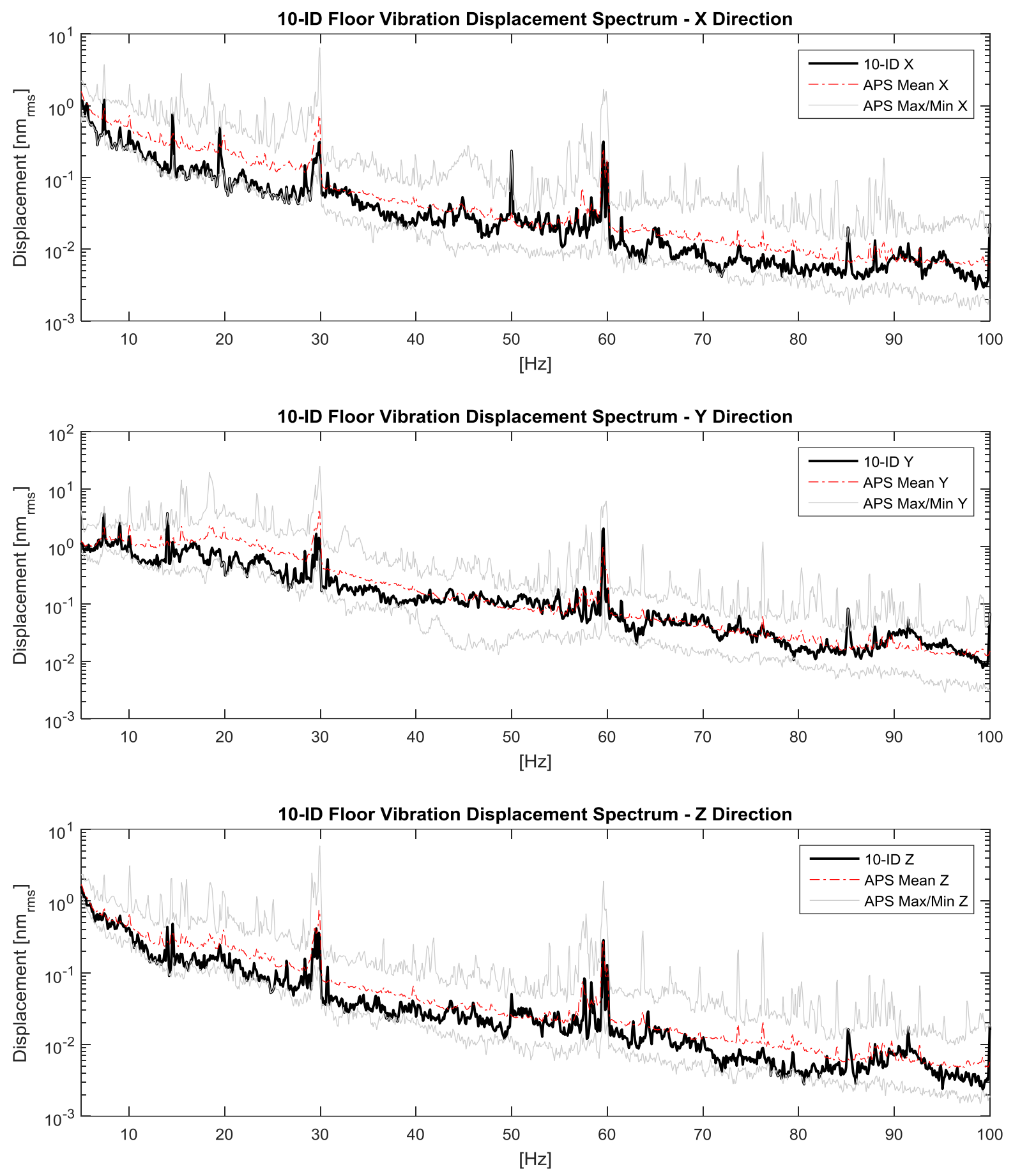

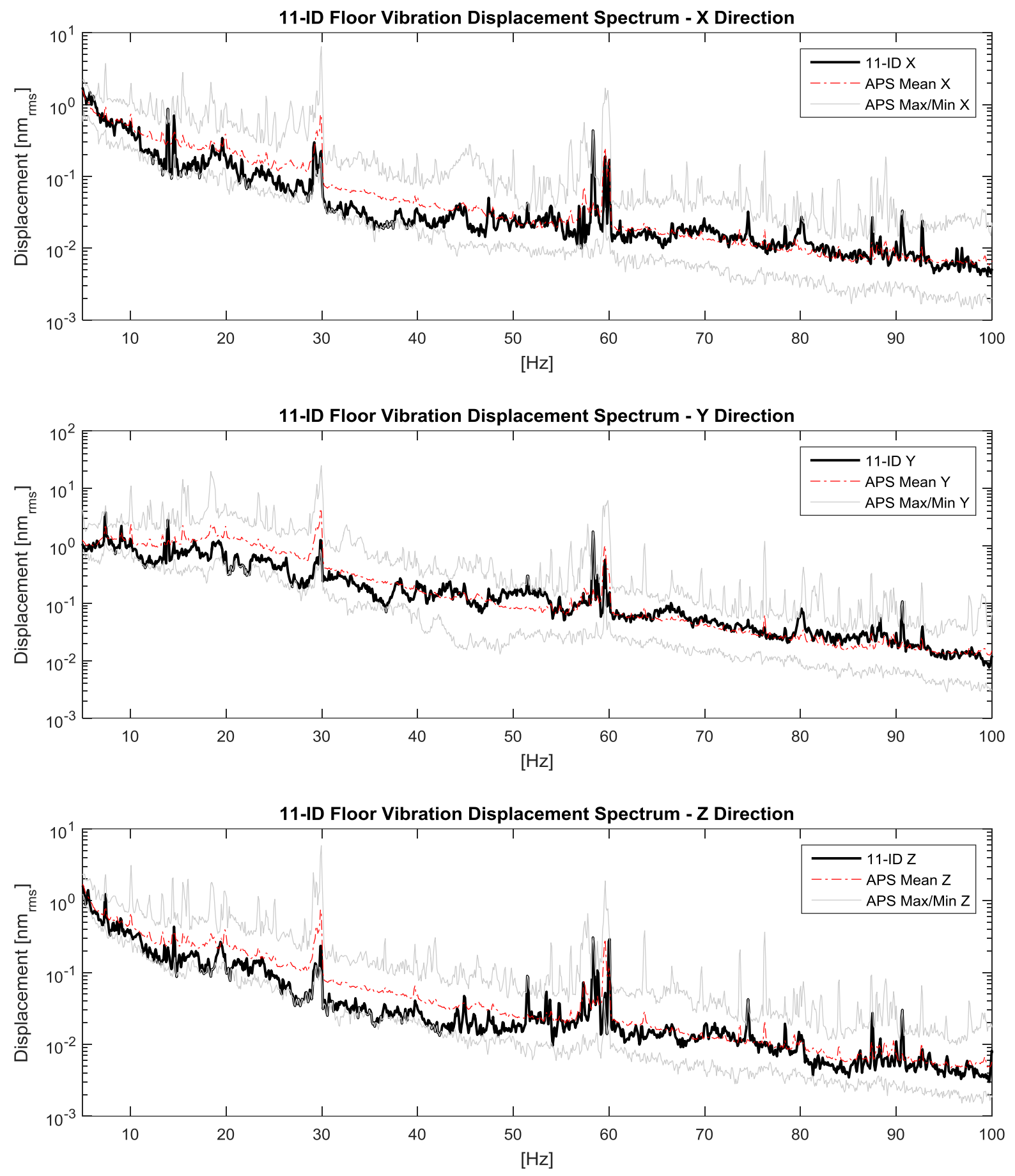

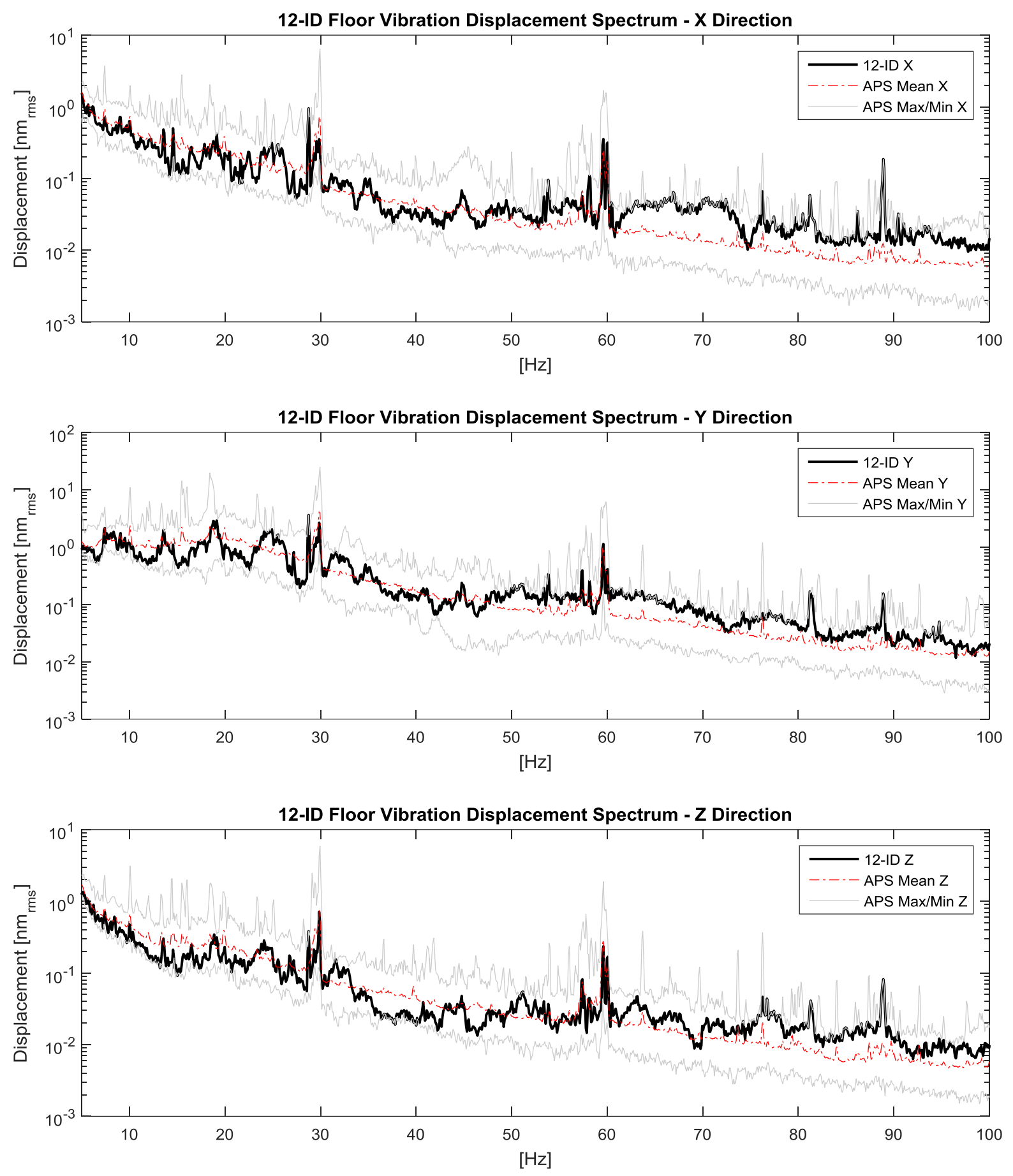

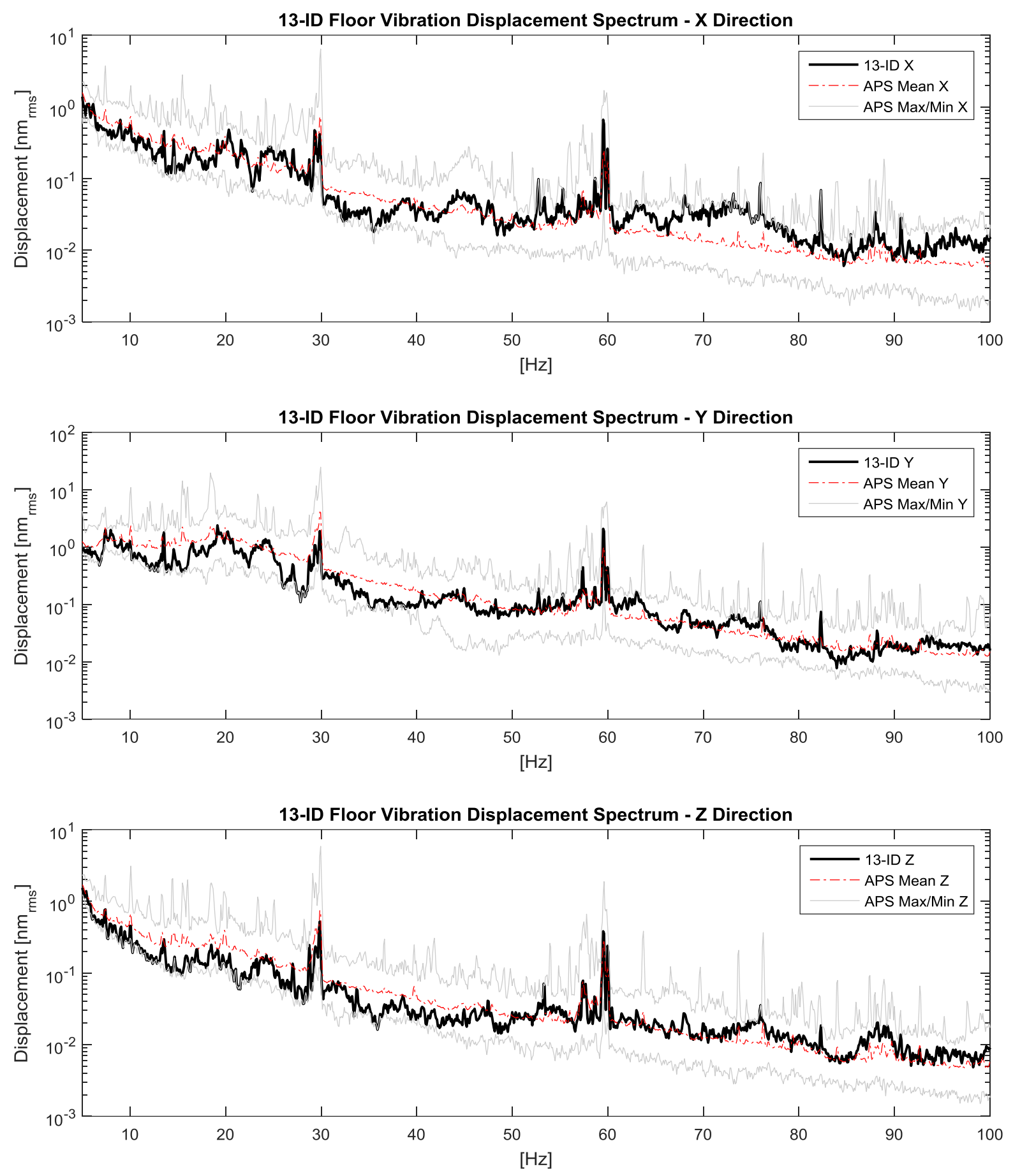

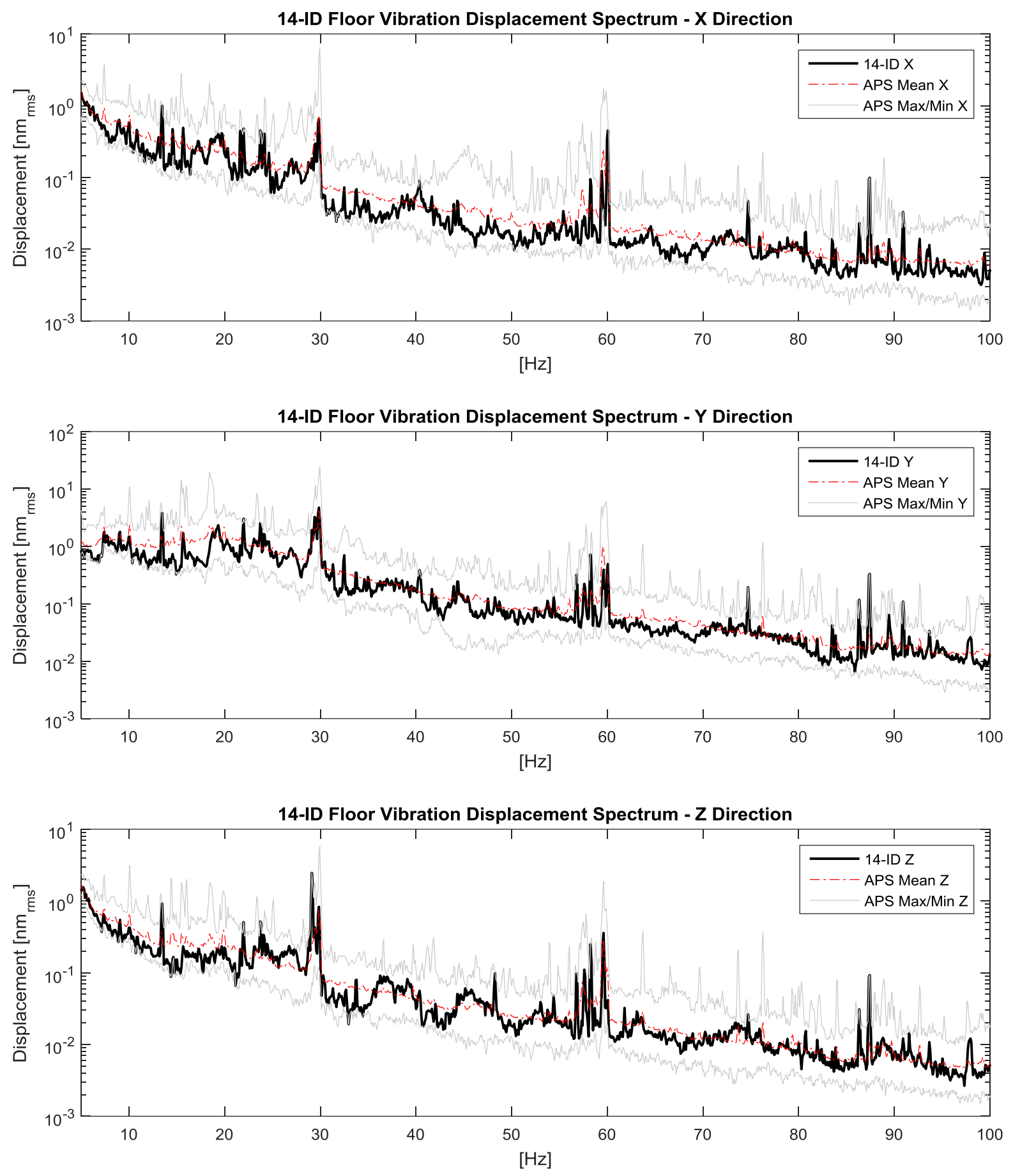

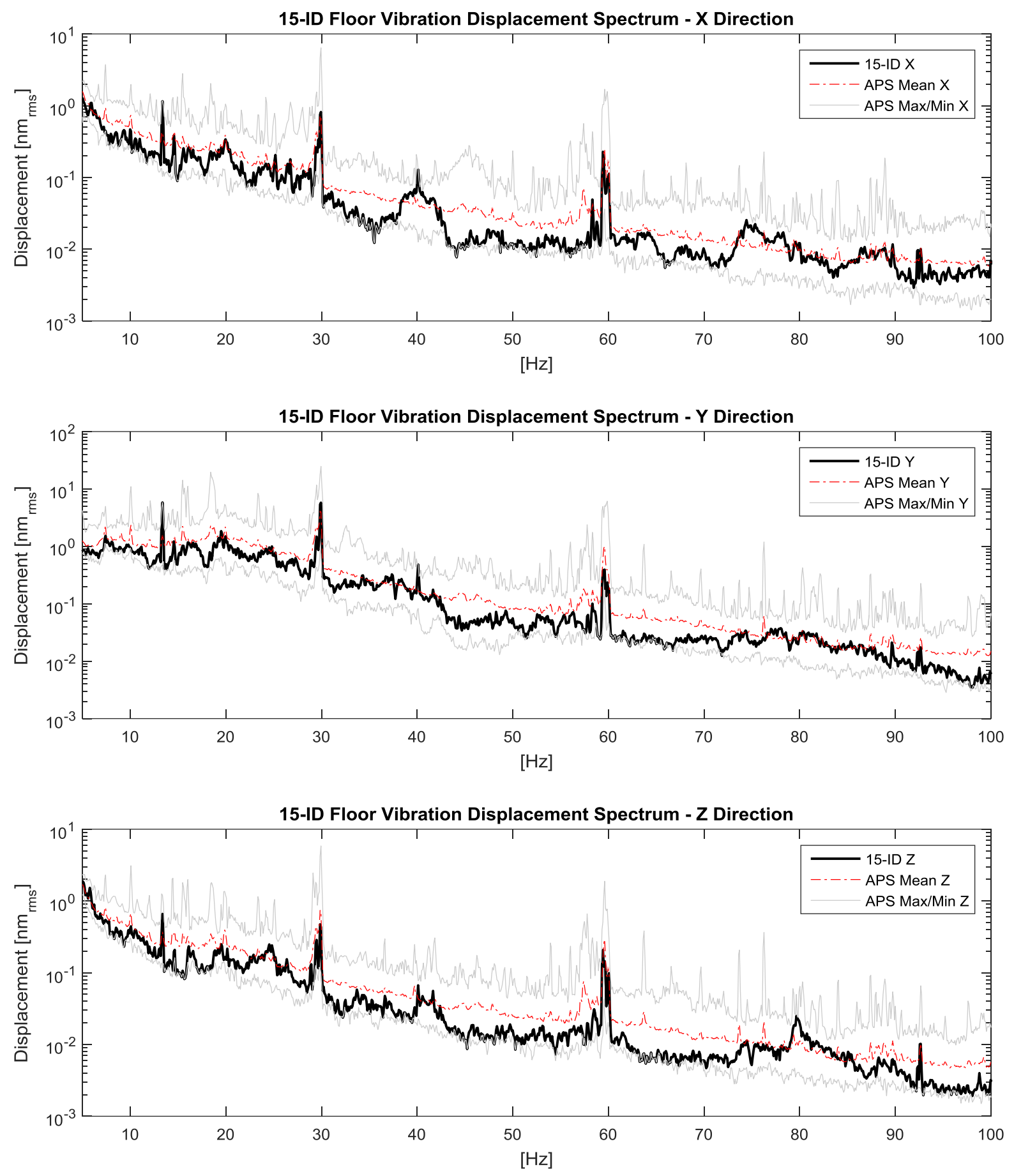

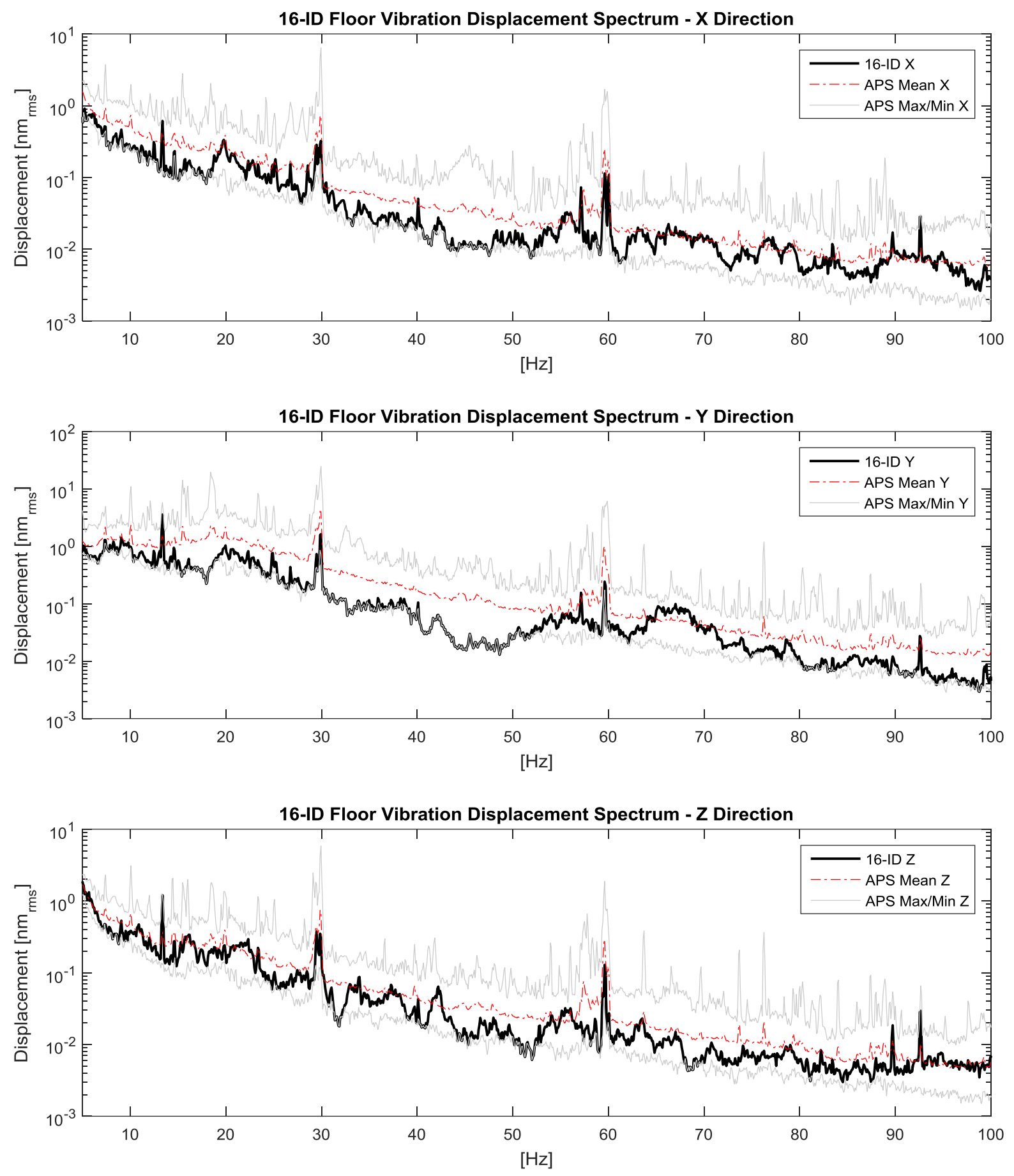

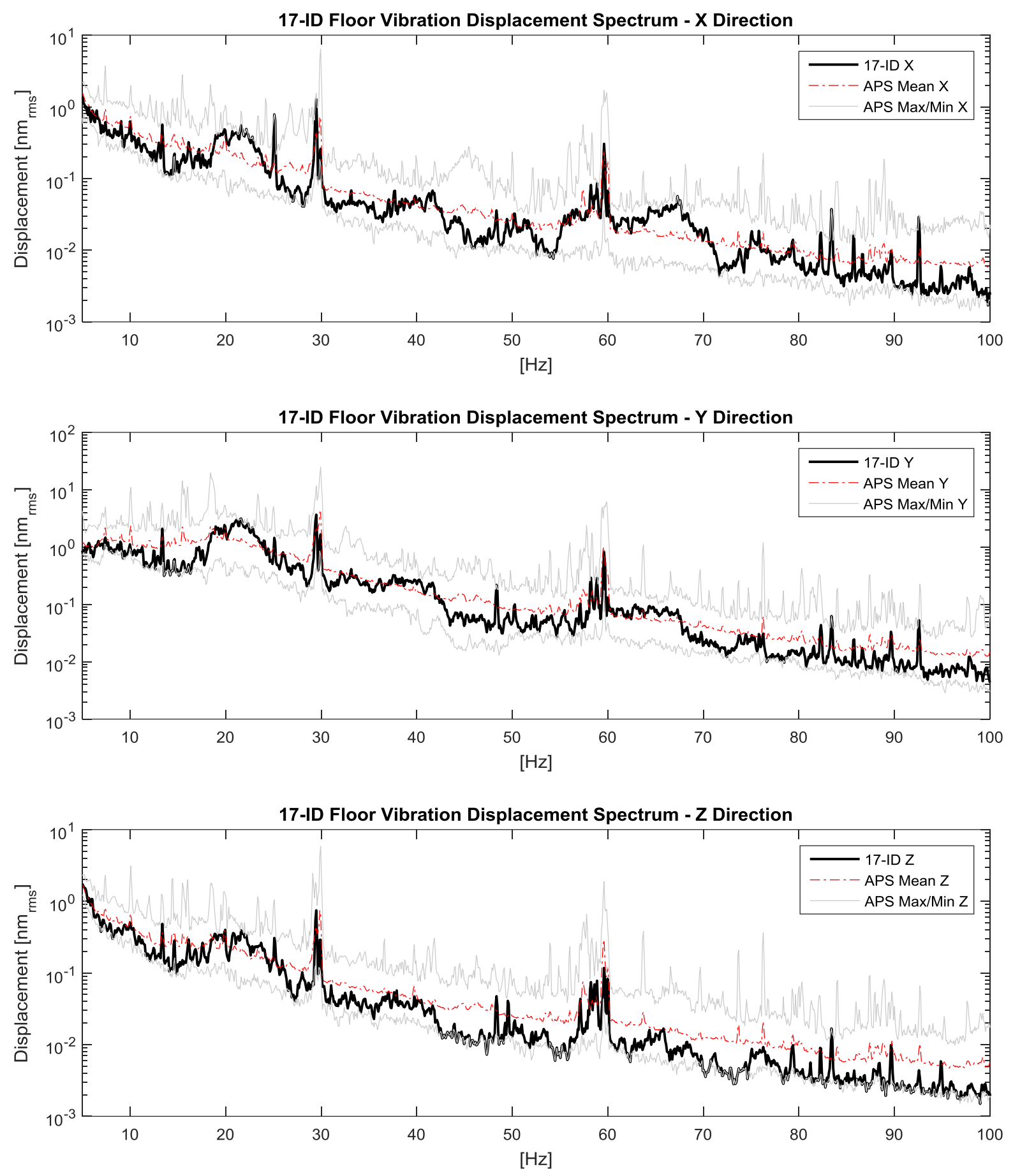

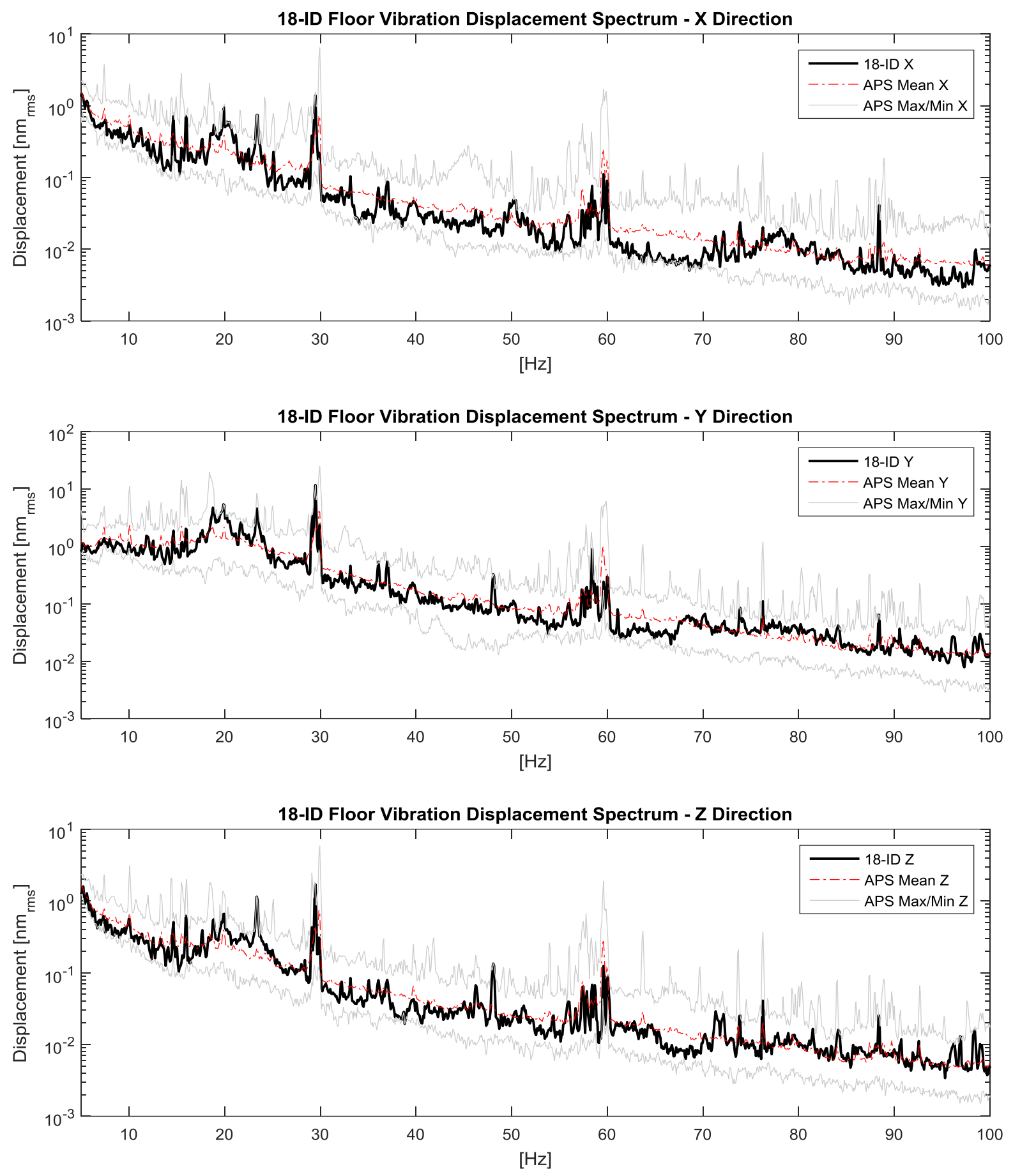

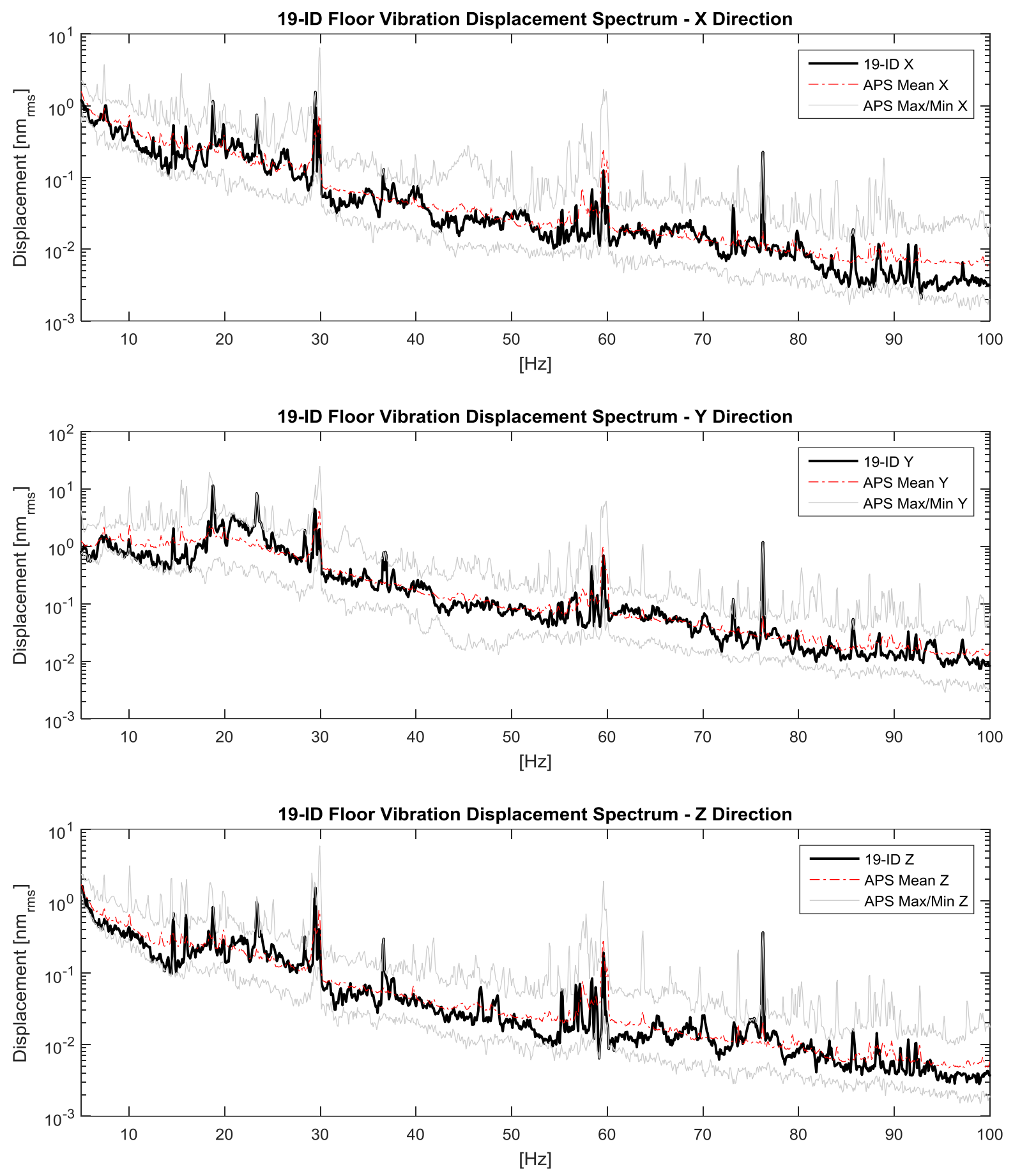

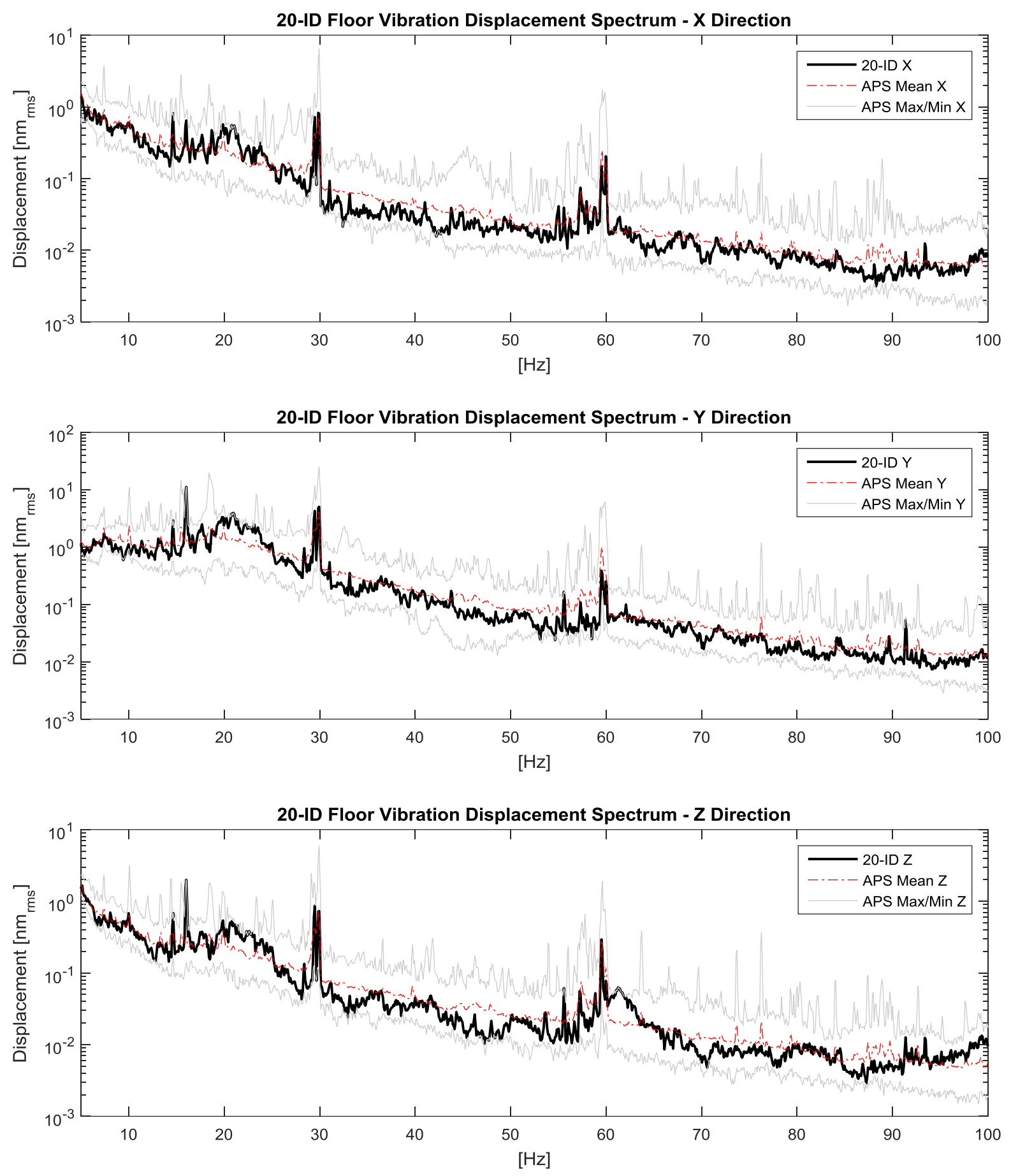

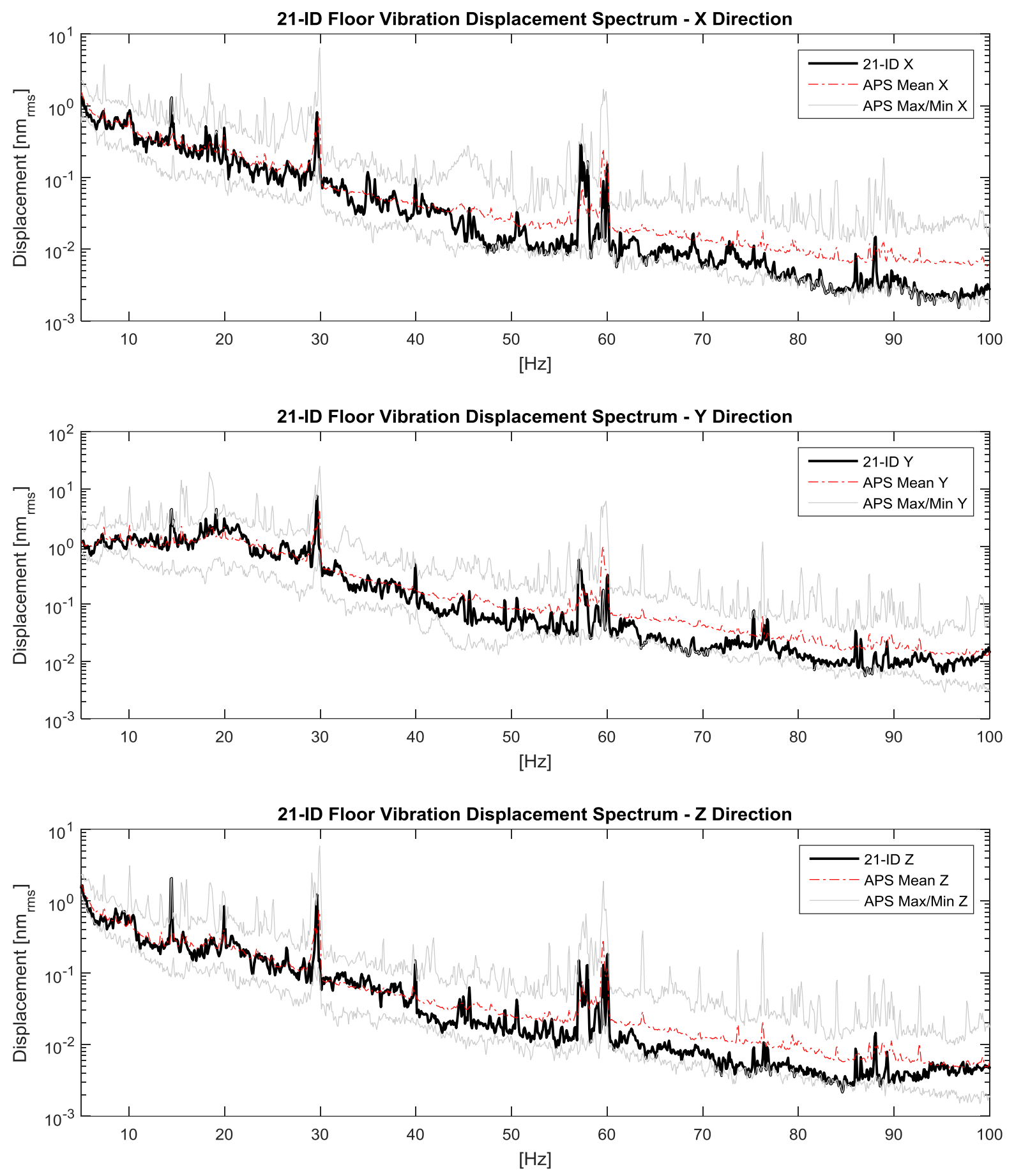

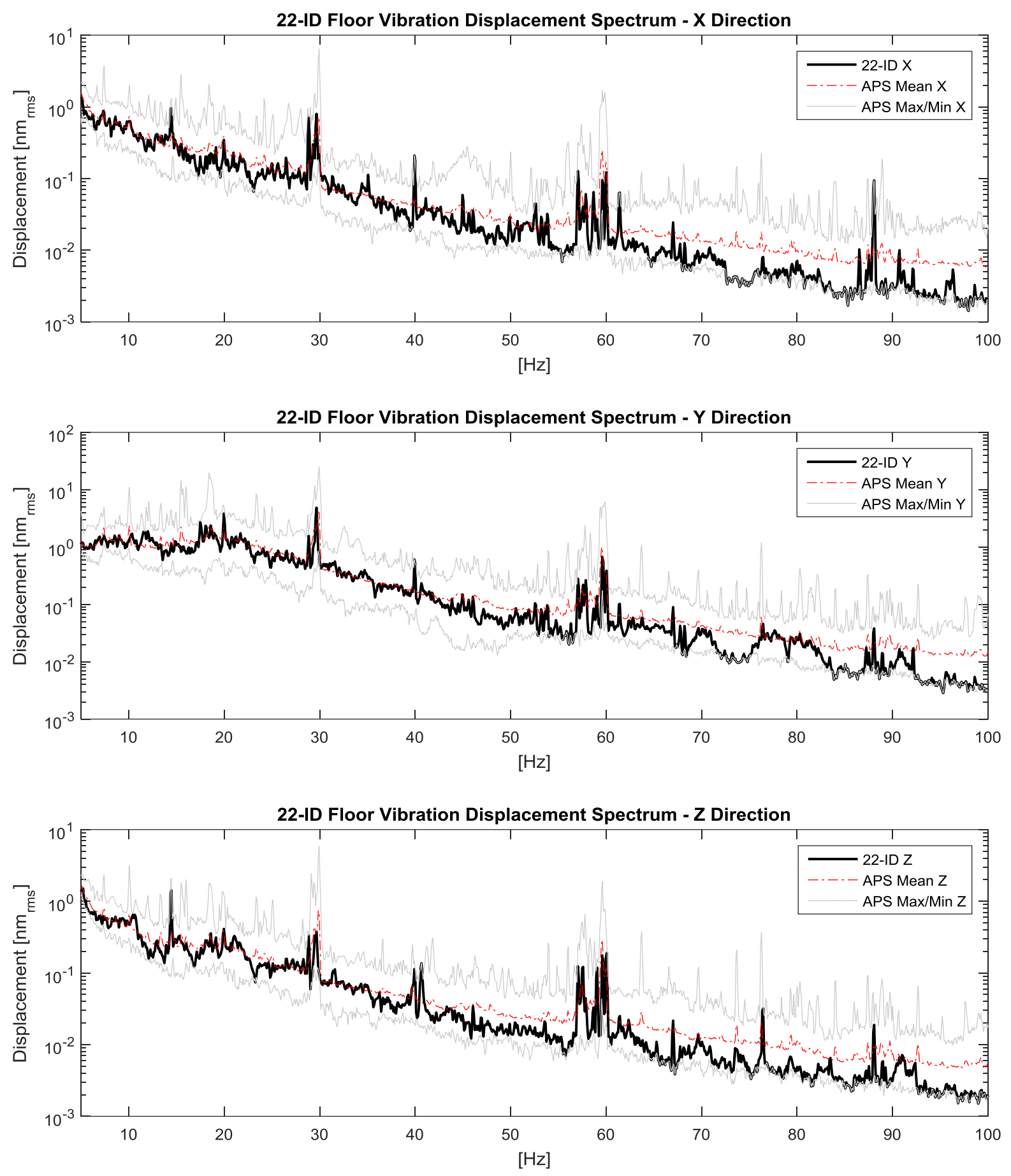

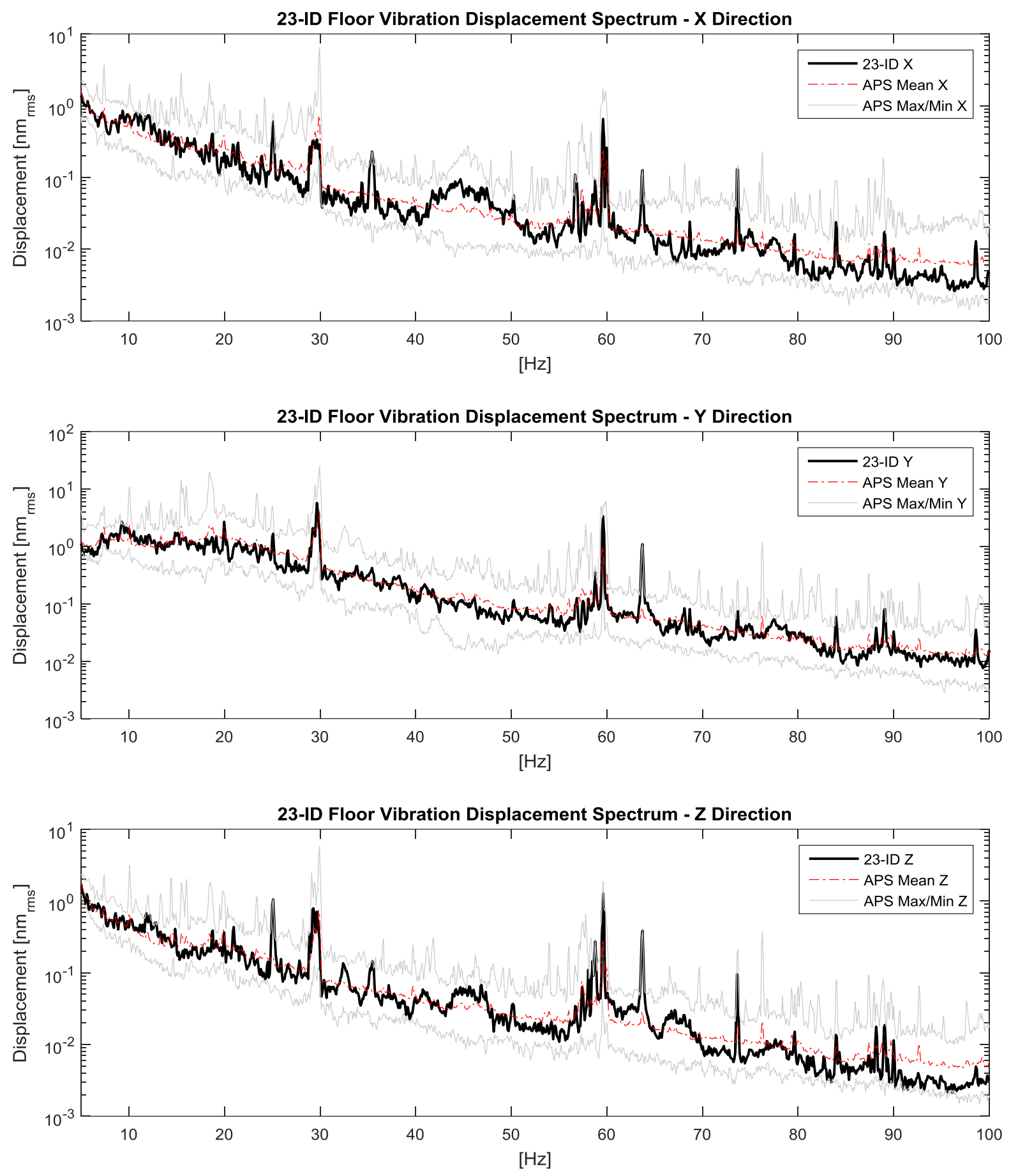

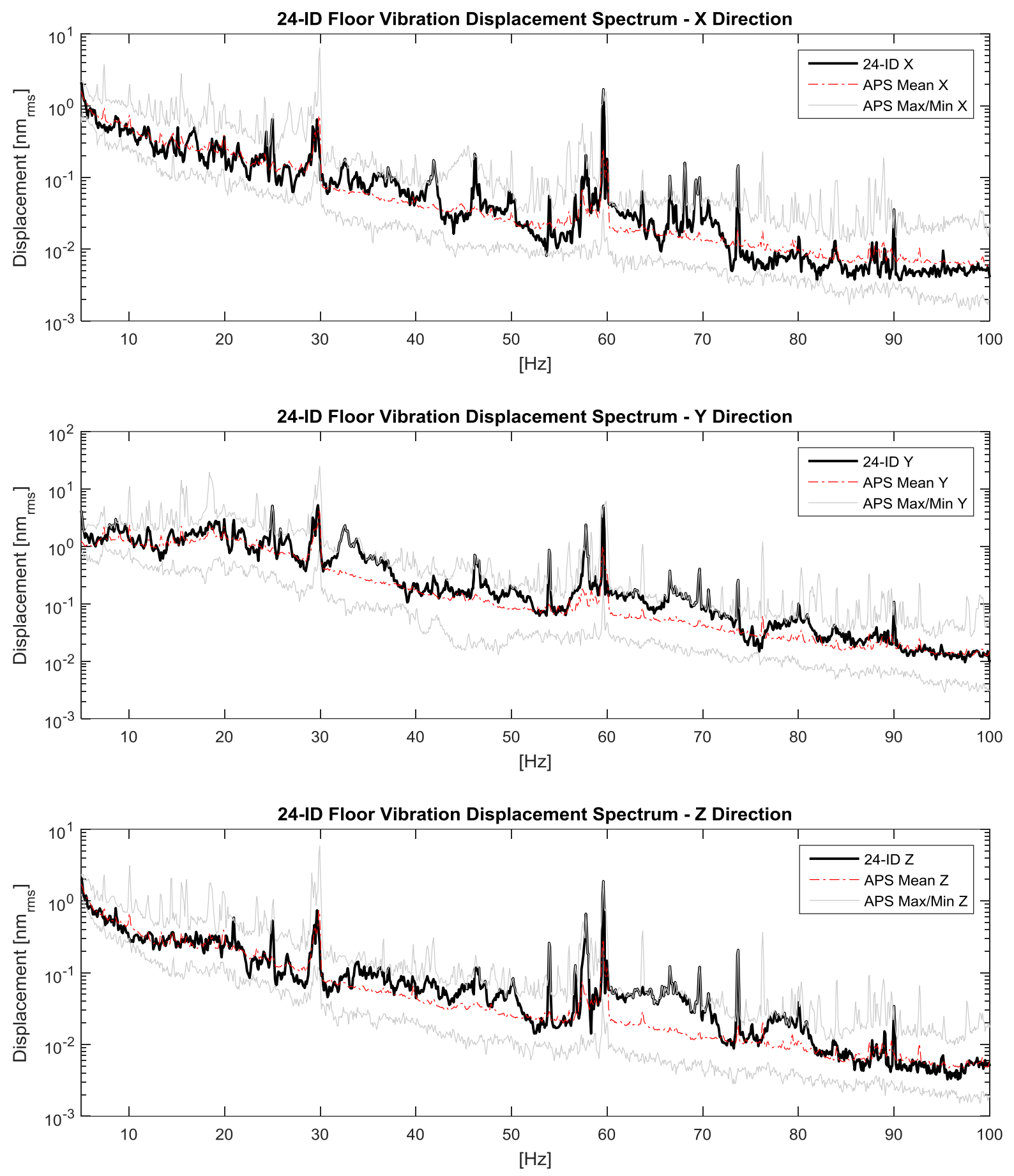

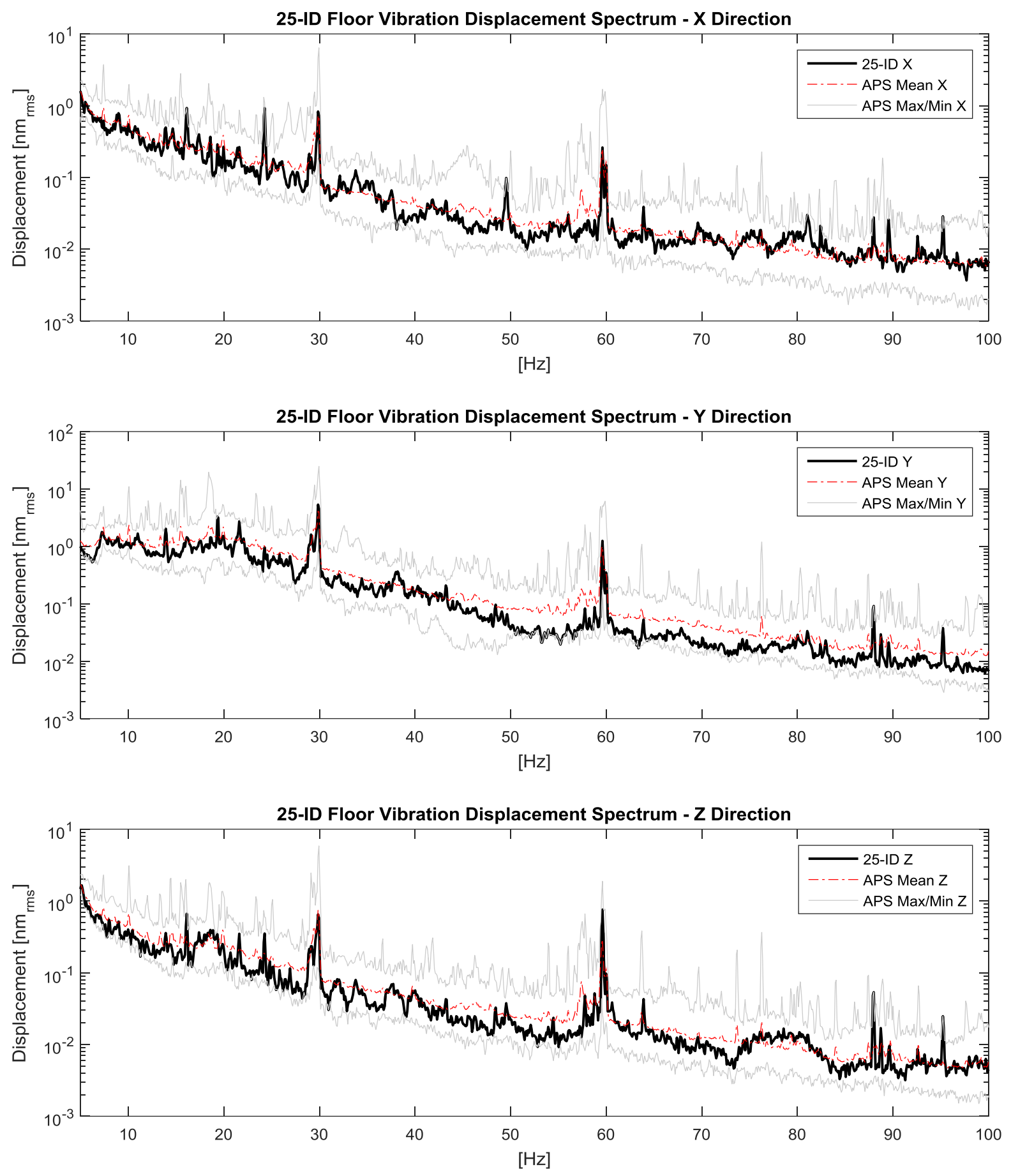

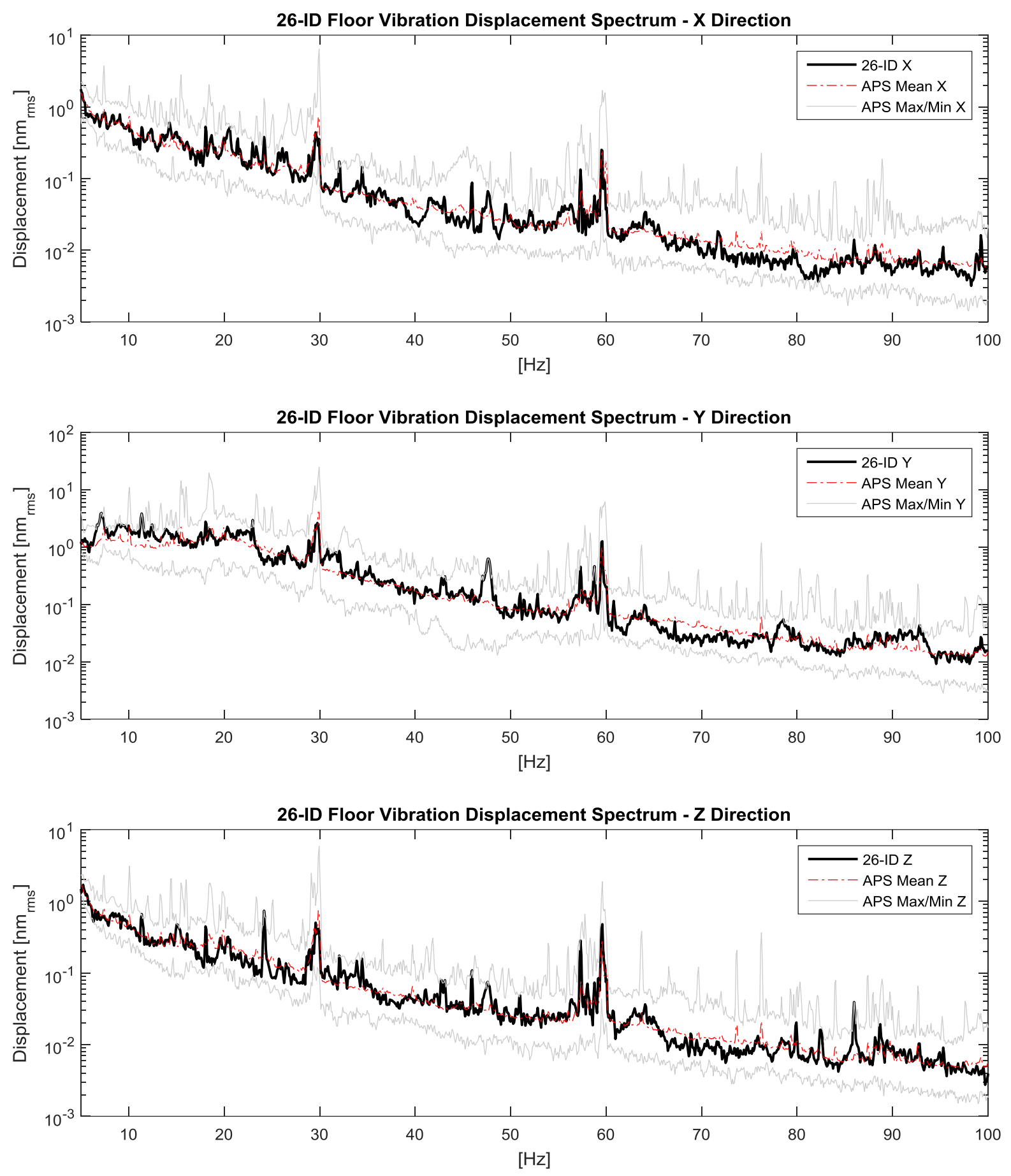

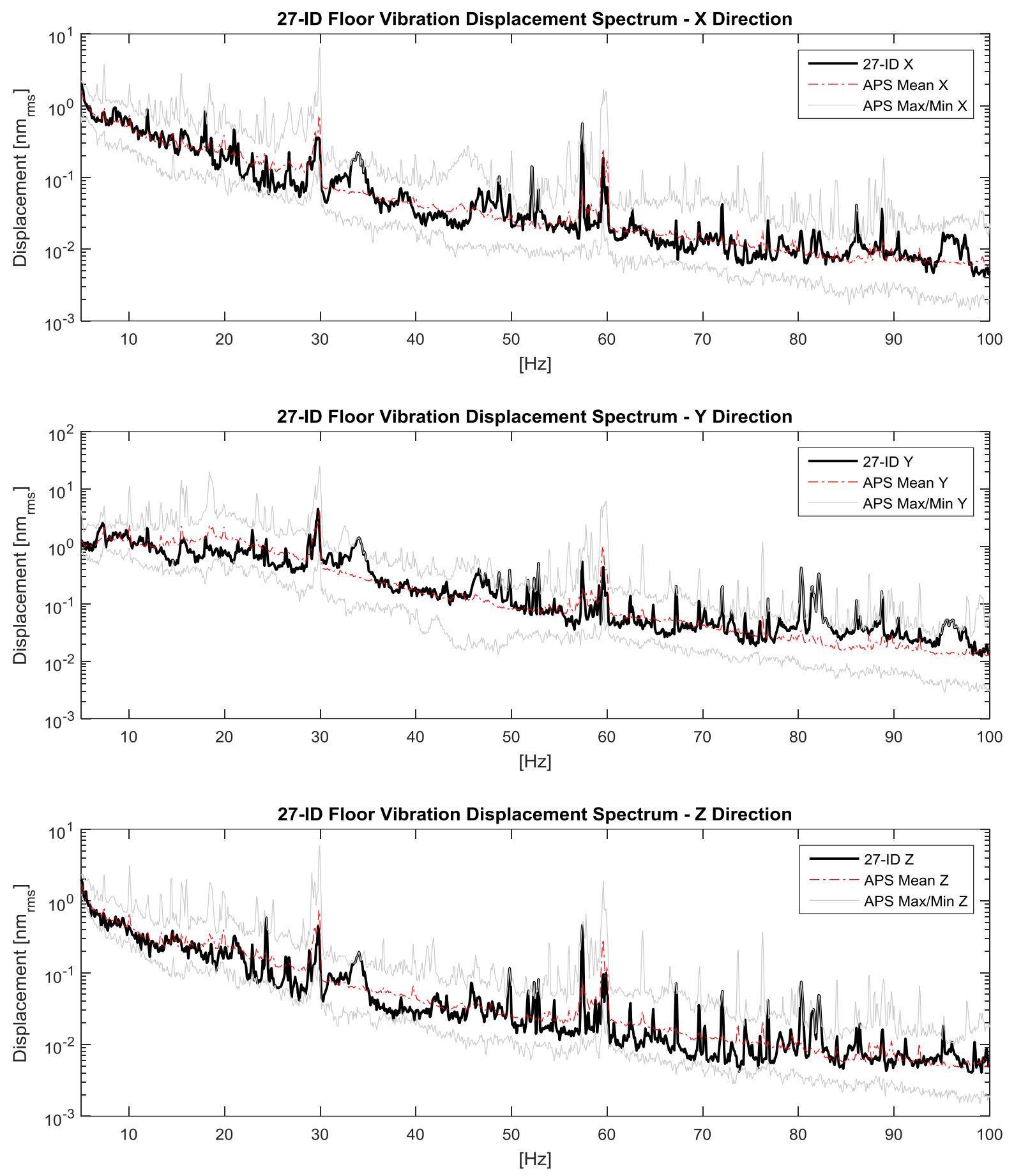

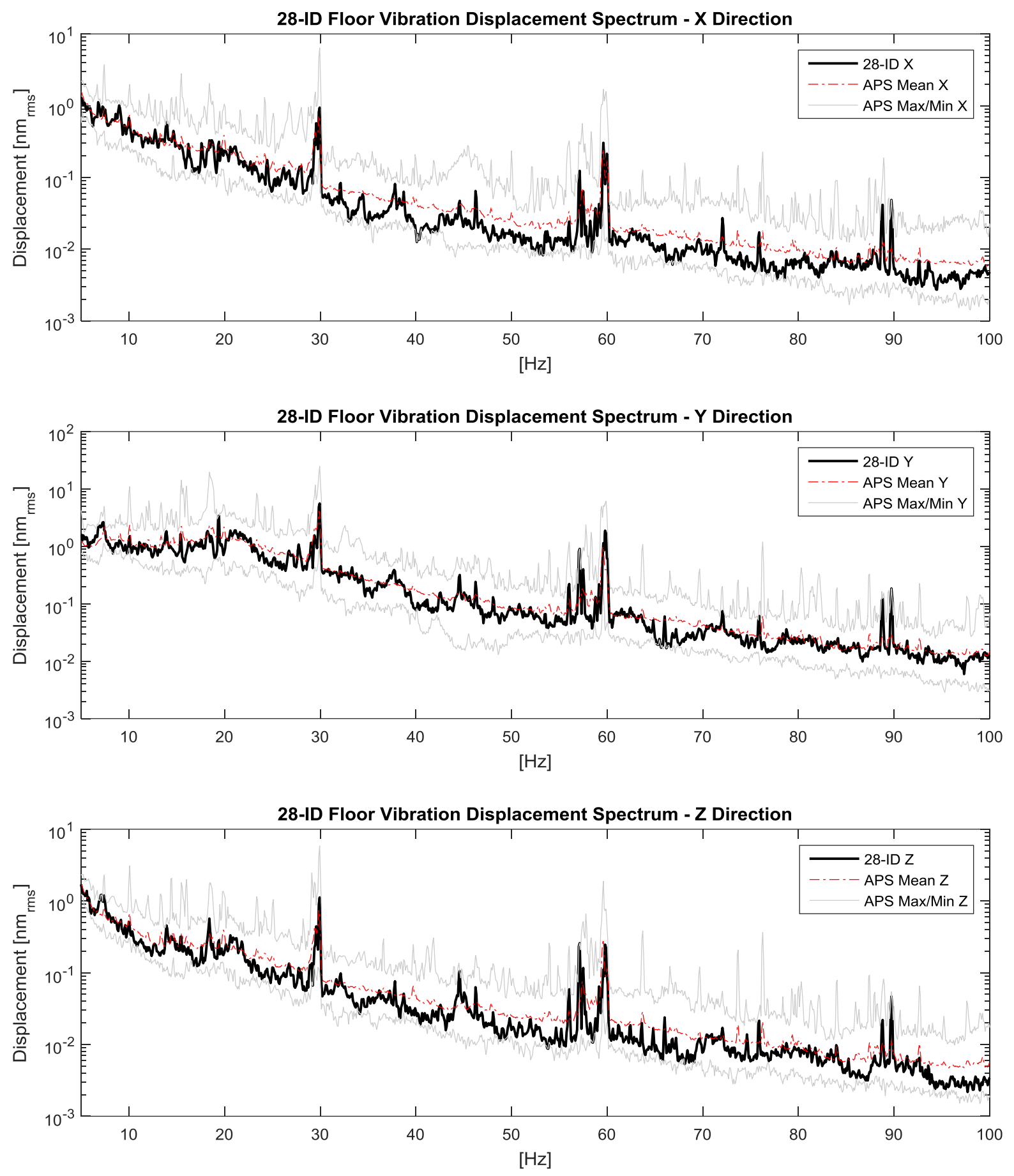

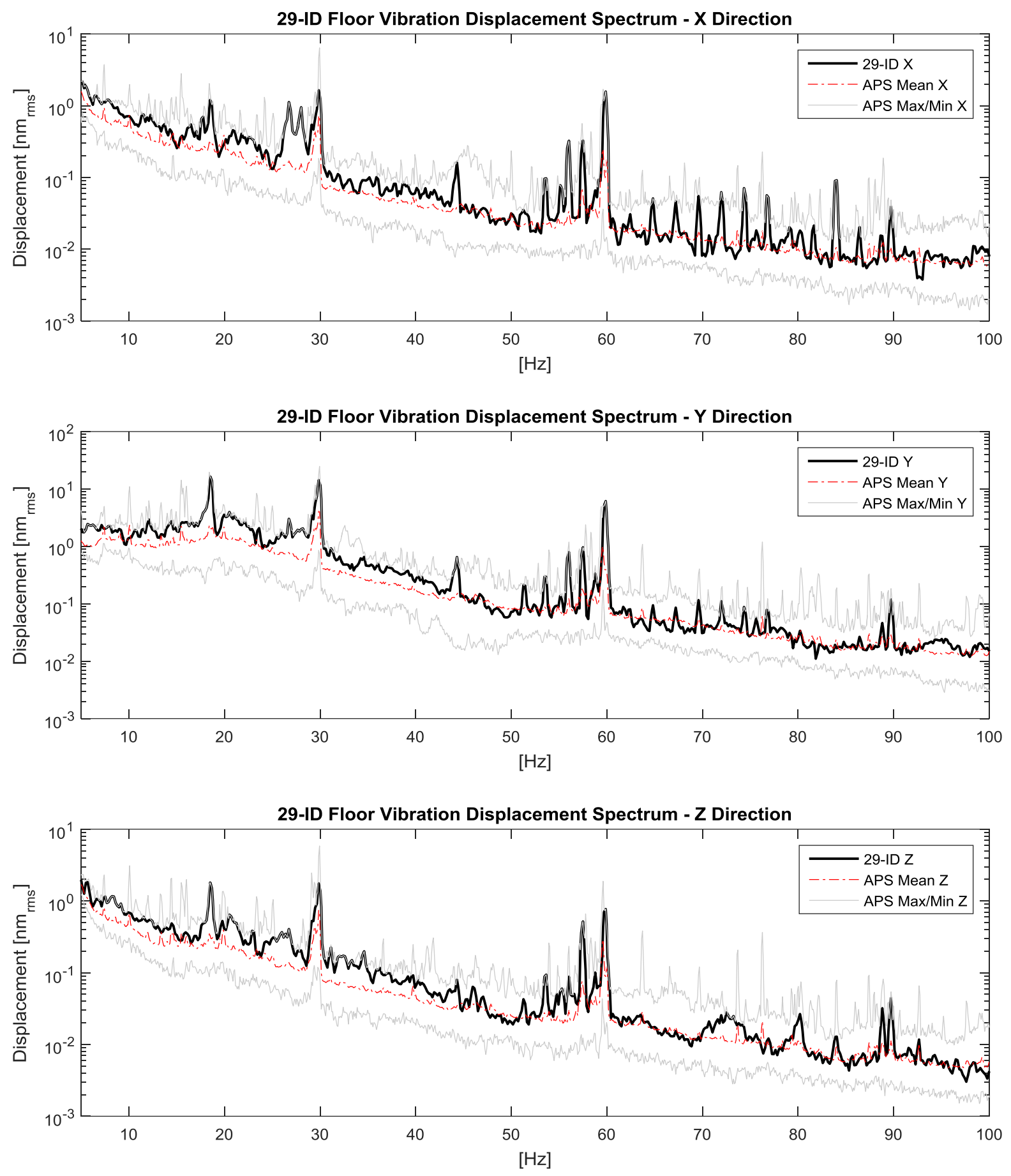

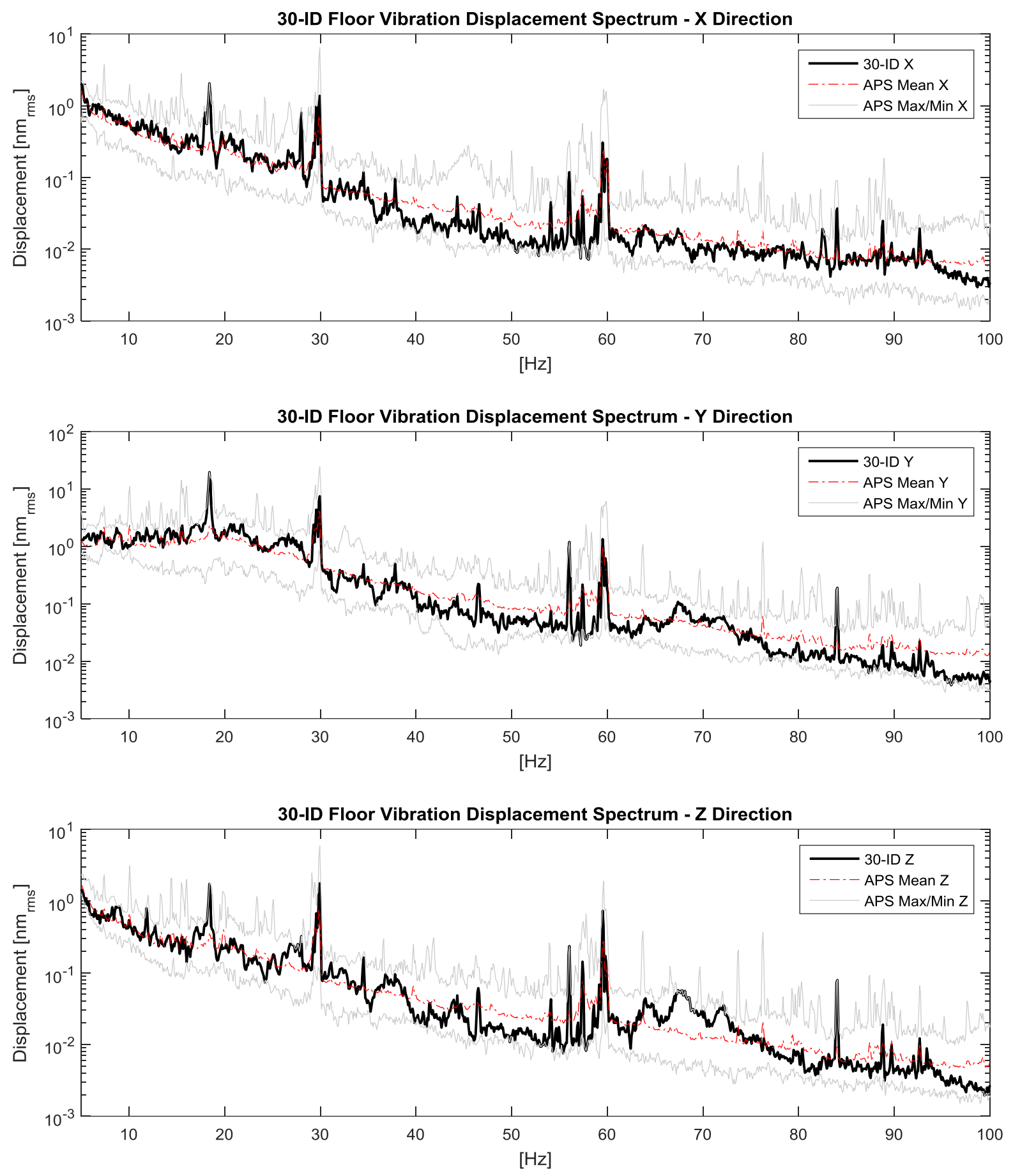

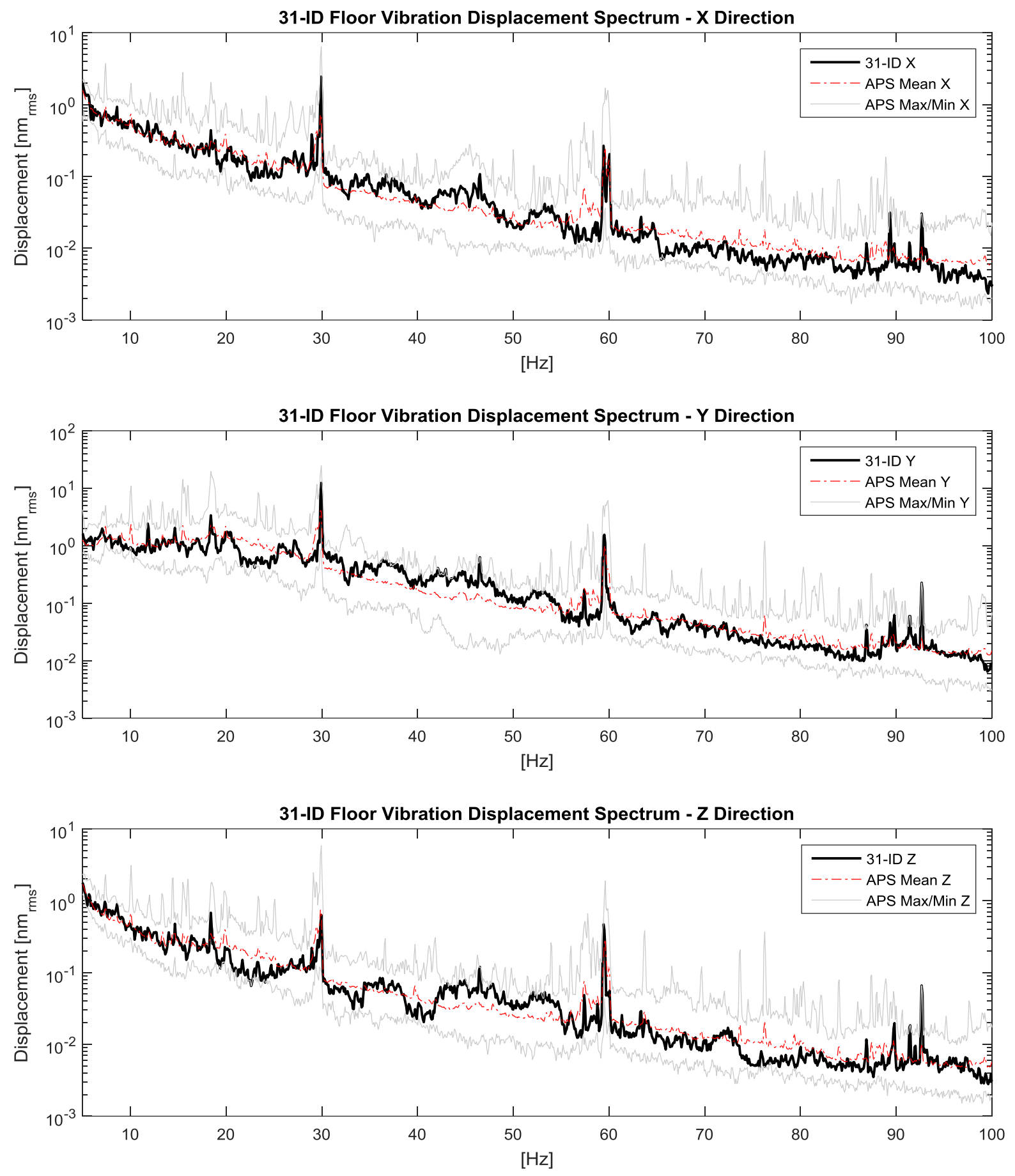

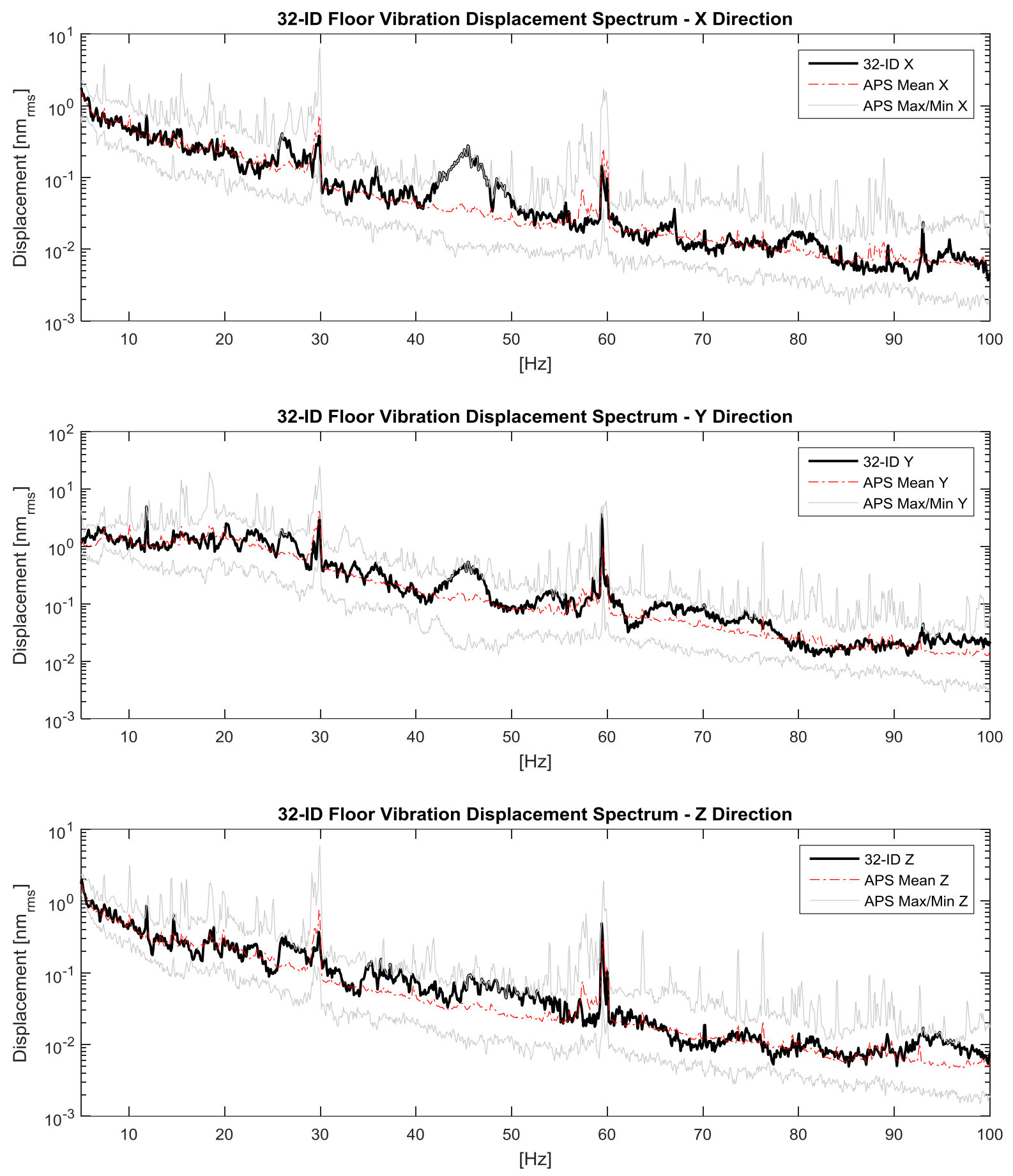

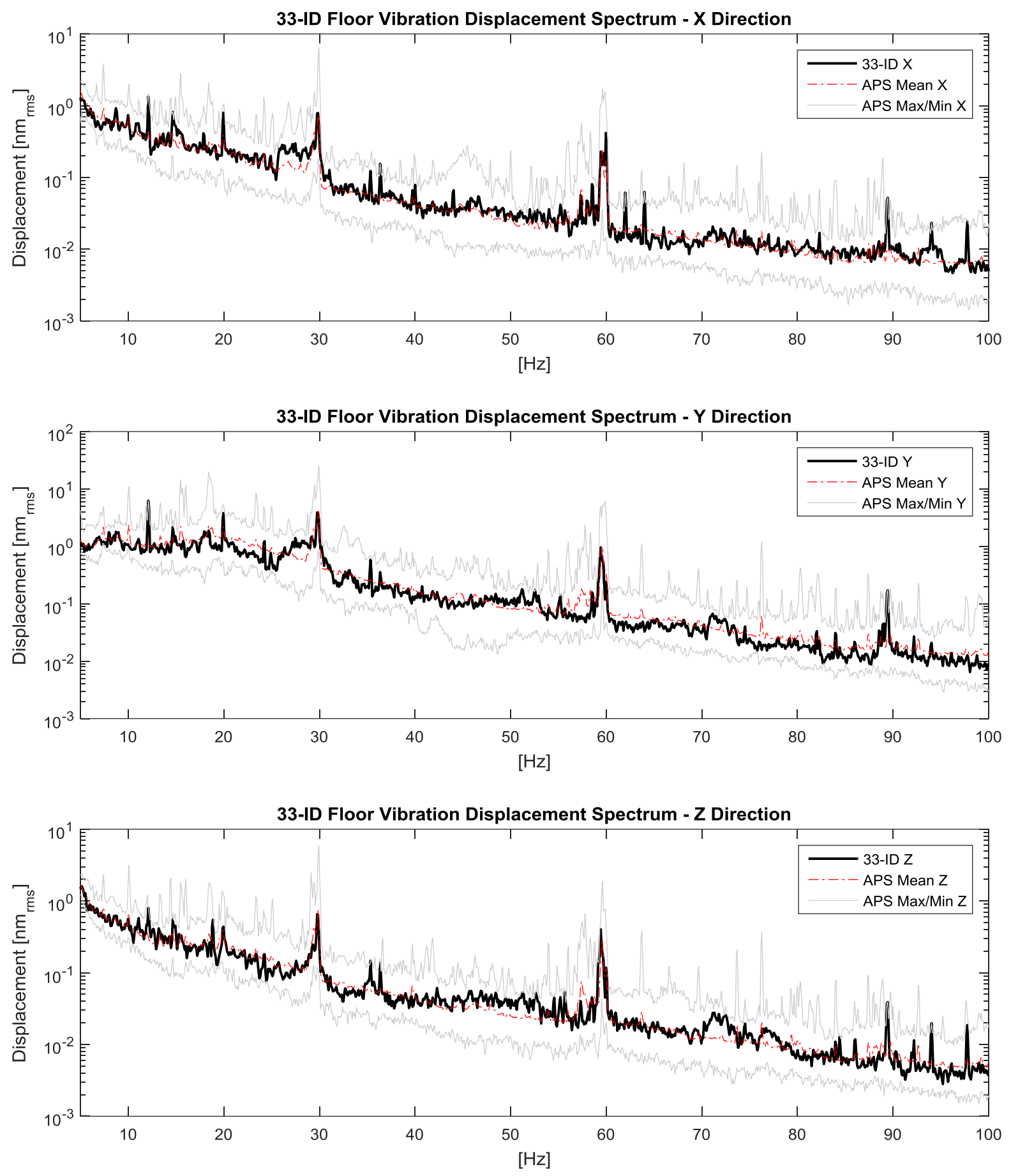

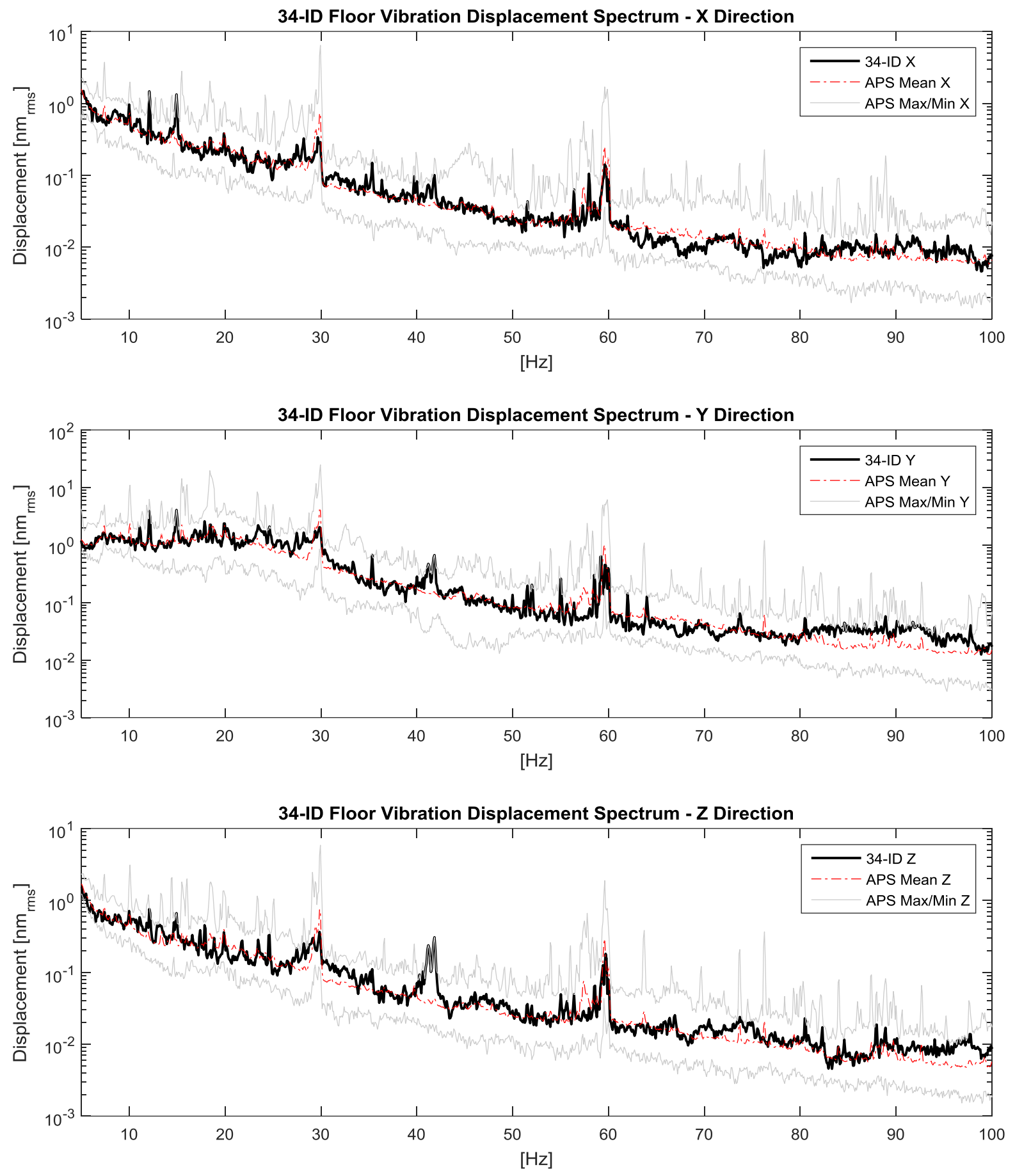

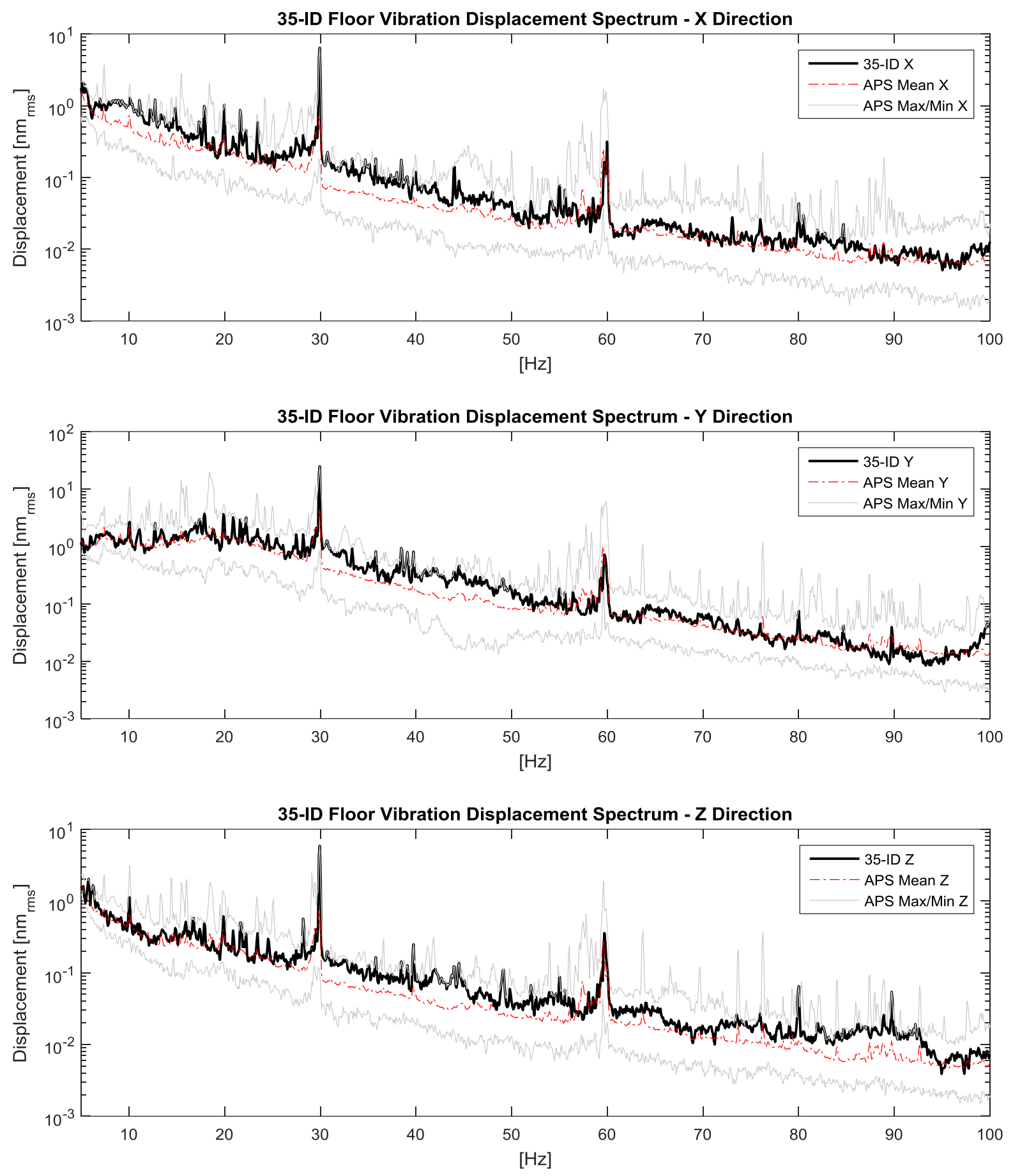